\title{
Doublecortin Associates with Microtubules Preferentially in Regions of the Axon Displaying Actin-Rich Protrusive Structures
}

\author{
Irina Tint, ${ }^{1,2}$ Daphney Jean, ${ }^{2}$ Peter W. Baas,,${ }^{2 *}$ and Mark M. Black ${ }^{1 *}$ \\ ${ }^{1}$ Department of Anatomy and Cell Biology, Temple University School of Medicine, Philadelphia, Pennsylvania 19140, and ${ }^{2}$ Department of Neurobiology and \\ Anatomy, Drexel University College of Medicine, Philadelphia, Pennsylvania 19129
}

\begin{abstract}
Here we studied doublecortin (DCX) in cultured hippocampal and sympathetic neurons during axonal development. In both types of neurons, DCX is abundant in the growth cone, in which it primarily localizes with microtubules. Its abundance is lowest on microtubules in the neck region of the growth cone and highest on microtubules extending into the actin-rich lamellar regions. Interestingly, the microtubule polymer richest in DCX is also deficient in tau. In hippocampal neurons but not sympathetic neurons, discrete focal patches of microtubules rich in DCX and deficient in tau are present along the axonal shaft. Invariably, these patches have actin-rich protrusions resembling those of growth cones. Many of the DCX/actin filament patches exhibit vigorous protrusive activity and also undergo a proximal-to-distal redistribution within the axon at average rates $\sim 2 \mu \mathrm{m} / \mathrm{min}$ and thus closely resemble the growth-cone-like waves described by previous authors. Depletion of DCX using small interfering RNA had little effect on the appearance of the growth cone or on axonal growth in either type of neuron. However, DCX depletion significantly delayed collateral branching in hippocampal neurons and also significantly lowered the frequency of actin-rich patches along hippocampal axons. Branching by sympathetic neurons, which occurs by growth cone splitting, was not impaired by DCX depletion. These findings reveal a functional relationship between the DCX/actin filament patches and collateral branching. Based on the striking resemblance of these patches to growth cones, we discuss the possibility that they reflect a mechanism for locally boosting morphogenetic activity to facilitate axonal growth and collateral branching.
\end{abstract}

\section{Introduction}

Different types of neurons in the CNS and PNS generate distinctive morphologies that are critical for their unique functions. Although environmental factors and cues are certainly important for molding neuronal morphology, it is noteworthy that various types of neurons when cultured identically retain many of their unique morphological characteristics, suggesting that aspects of neuronal morphology are endogenously determined. The mechanisms that generate neuronal morphology ultimately converge on the cytoskeleton to establish the patterns of cytoskeletal organization that constitute the structural basis of neuronal form. A growing body of evidence indicates that these patterns result from a coordinated interaction of microtubules and actin filaments. This is seen particularly well in the process of collateral branch formation (Dent and Kalil, 2001) and in the growth cone, which generates the motility required for axonal elongation and

Received July 15, 2009; accepted July 24, 2009.

This work was supported by grants from the National Institutes of Health and the National Science Foundation (P.W.B.). I.T. is supported by a National Institutes of Health postdoctoral training grant. We thank Orly Reiner of the Weizmann Institute and Christopher Walsh of the Beth Israel Deaconess Medical Center for providing antibodies. We thank Wenqian Yu of Drexel University College of Medicine and Theresa Slaughter of Temple University School of Medicine for assistance and support.

*P.W.B. and M.M.B. contributed equally to this work.

Correspondence should be addressed to Mark Black, Department of Anatomy and Cell Biology, Temple University School of Medicine, 3400 North Broad Street, Philadelphia, PA 19140. E-mail: mark.black@temple.edu. DOI:10.1523/JNEUROSCI.3399-09.2009

Copyright $\odot 2009$ Society for Neuroscience ～0270-6474/09/2910995-16\$15.00/0 pathfinding (Dent and Gertler, 2003). There is an ever growing list of proteins that can theoretically impact both cytoskeletal elements and thereby integrate them, including structural proteins such as MAP1b (Bouquet et al., 2007), motor proteins such as cytoplasmic dynein (Myers et al., 2006), and signaling proteins such as small G-proteins (Grabham et al., 2003).

In the present study, we have focused on a protein called doublecortin (DCX), which has been shown in vitro to influence microtubule stability, bundling, and protofilament number (Francis et al., 1999; Gleeson et al., 1999; Horesh et al., 1999; Moores et al., 2004, 2006). In addition, DCX can interact with actin filaments either directly or indirectly via neurabin II (Tsukada et al., 2003, 2005, 2006; Shmueli et al., 2006). A potential role for DCX as an integrator of crosstalk between cytoskeletal systems is further supported by observations that DCX can be phosphorylated by a variety of kinases and can form complexes with known signaling proteins (Gdalyahu et al., 2004; Graham et al., 2004; Schaar et al., 2004; Tanaka et al., 2004; Shmueli et al., 2006; Tsukada et al., 2006).

Many of the proposed axonal functions of DCX, particularly with regard to microtubules and actin filaments, lead to specific predictions about its detailed localization and subcellular associations. Although several groups have shown that DCX is present in growing axons, in which is it enriched distally in the growth cone (Friocourt et al., 2003; Gdalyahu et al., 2004; Schaar et al., 2004), the data do not reveal whether DCX is associated with microtubules, actin filaments, or other structures, nor do they 
reveal the relative abundance of DCX on these structures in specific domains of the growth cone. Here, we sought to thoroughly analyze the distribution of DCX in both hippocampal and sympathetic neurons, as well as the effects on axonal morphology of depleting DCX with small interfering RNA (siRNA). Our results reveal that the microtubule association of DCX is tightly regulated in growing axons and that the actin system participates in this regulation.

\section{Materials and Methods}

Materials. Culture dishes in which glass coverslips had been fixed to the bottom over a hole of $14 \mathrm{~mm}$ were obtained from Matek. Culture media were obtained from Invitrogen. Supplements for culture media were obtained from Invitrogen, except for BSA, which was obtained from Calbiochem, and fetal bovine serum, which was obtained from HyClone Laboratories. Other reagents were obtained from Sigma-Aldrich unless otherwise indicated.

Cell culture. Experiments used either primary cultures of sympathetic or hippocampal neurons from rat. Sympathetic neurons were dissociated from the superior cervical ganglia of 1 - to 3 -d-old rat pups using sequential treatments with collagenase and trypsin, followed by trituration. Dissociated neurons were plated onto glass coverslips coated with polylysine and subsequently laminin as described previously (Brown et al., 1992), except that laminin was used at a final concentration of $25 \mu \mathrm{g} / \mathrm{ml}$. For studies on early stages of axonal growth, cells were plated on polylysine alone. With this substrate, neurons attach but fail to extend axons during the first couple of days in culture. However, rapid axonal growth can be triggered by addition of laminin to the culture medium (Rivas et al., 1992; Slaughter et al., 1997; Yu et al., 2001). Hippocampal neurons were dissociated from hippocampi dissected from $18 \mathrm{~d}$ rat fetuses and plated onto polylysine-coated glass coverslips as described by Yu et al. (2008). Culture media were modified to contain astrocyte-conditioned media using the procedure of Kaech and Banker (2006). To acutely depolymerize actin filaments, cultures of neurons were treated with $25 \mu \mathrm{M}$ latrunculin (Invitrogen) for 3 or $10 \mathrm{~min}$.

RNA interference. siRNA-mediated depletion of DCX was used to evaluate its role in axonal development and cytoskeletal organization. siRNA was introduced into dissociated neurons using the Amaxa nucleofection device as described previously (Hasaka et al., 2004; He et al., 2005; Myers et al., 2006). Four different siRNA duplexes were designed against different regions of the DCX sequence using the custom SMARTpool siRNA service of Dharmacon (rat cytoplasmic DCX; GenBank accession number NM_053379). The nonspecific duplex III from Dharmacon was used as control. siRNA was dissolved to $200 \mu \mathrm{M}$, aliquotted, and stored at $-20^{\circ} \mathrm{C}$. siRNA concentration at nucleofection was $18 \mu \mathrm{M}$. Initial experiments using immunostaining and immunoblotting indicated that DCX levels declined progressively over time, reaching $\geq 80 \%$ depletion in most neurons by $3 \mathrm{~d}$ after nucleofection. Accordingly, nucleofected neurons were plated at high density onto $35 \mathrm{~mm}$ plastic culture dishes and maintained for $3 \mathrm{~d}$, at which time they were dissociated by brief trypsin treatment and then replated onto glass coverslips as described above ( $\mathrm{He}$ et al., 2005; Myers et al., 2006; Qiang et al., 2006). In this way, neurons were challenged to regrow axons after DCX was reduced by $\geq 80 \%$.

Immunofluorescence procedures. Two procedures were used to fix cells for immunostaining. In a limited number of experiments, cells were fixed without extraction by immersion in cold $\left(-20^{\circ} \mathrm{C}\right)$ methanol for $10 \mathrm{~min}$ (Roy et al., 2000). Fixed cells were rehydrated with PBS and then stained as described below. For most experiments, we used a combined fixation/ extraction procedure in which cultures were rinsed twice with PBS and then fixed by immersion for 10 min in PEM (100 mM PIPES, 5 mM EGTA, and $2 \mathrm{mM} \mathrm{MgCl}_{2}, \mathrm{pH} 6.8$ ) containing $0.3 \%$ glutaraldehyde, $0.1 \%$ Igepal CA-630, $10 \mu \mathrm{m}$ taxol, and $1 \mu \mathrm{g} / \mathrm{ml}$ phalloidin. Cells were then rinsed extensively in PBS, $\mathrm{pH}$ 7.4, further permeabilized by incubation with $0.5 \%$ Triton X-100 in PBS for $15 \mathrm{~min}$, and then treated twice with 10 $\mathrm{mg} / \mathrm{ml}$ sodium borohydride in PBS, $7 \mathrm{~min}$ each time, to quench glutaraldehyde-related autofluorescence. For staining, samples fixed by either method were blocked by incubation for $30 \mathrm{~min}$ with $10 \mathrm{mg} / \mathrm{ml}$ BSA plus $10 \%$ normal donkey serum in PBS and then incubated with primary antibodies, diluted in PBS for $60 \mathrm{~min}$ at room temperature, rinsed extensively with PBS, and reblocked for at least $10 \mathrm{~min}$ before incubation with secondary antibodies, diluted in PBS, for $45 \mathrm{~min}$ at room temperature. After extensive rinsing in PBS, samples were mounted in a medium that reduces photobleaching $(0.212 \% \mathrm{~N}$-propylgallate in $90 \%$ glycerol and $10 \%$ PBS).

Methanol-fixed cells were double stained for microtubules and DCX. For these analyses, cells were incubated first with antibody to DCX, rinsed with $\mathrm{PBS}$, and then incubated with antibody to tubulin, rinsed extensively, and then incubated simultaneously with secondary antibodies. Cells fixed/extracted with glutaraldehyde were triple stained to reveal microtubules, DCX, and actin or tau, another microtubule-associated protein present in axons. To triple stain for microtubules, DCX, and actin filaments, cells were incubated first with antibody to DCX, rinsed with PBS, incubated with antibody to tubulin, rinsed extensively, and then incubated simultaneously with secondary antibodies and rhodaminelabeled phalloidin. To triple stain for microtubules, DCX, and tau, cells were incubated first with the tau antibody (tau 5') (Black et al., 1996), rinsed, incubated with DCX antibody, rinsed again, and incubated with tubulin antibody, rinsed extensively, and then incubated simultaneously with secondary antibodies. The following antibodies were used for the present experiments. Microtubules were revealed using a mouse monoclonal antibody against $\alpha$-tubulin [DM1 $\alpha$, provided by Dr. V. Gelfand, University of Illinois, Urbana, IL (Blose et al., 1984)]. The tau $5^{\prime}$ antibody is a rabbit polyclonal raised against the $\mathrm{N}$-terminal $\sim 100$ aa of rat tau and is specific for tau as assessed by immunoblotting against extracts of brain and cultured neurons (Black et al., 1996). To reveal DCX, most experiments used the C-18 antibody purchased from Santa Cruz Biotechnology; this is a peptide antibody raised in goats, and we used it typically at a dilution of 1:250 $(4 \mu \mathrm{g} / \mathrm{ml})$. In a limited number of experiments, we also used rabbit polyclonal antibodies against DCX generously provided by Dr. Orly Reiner (Department of Molecular Genetics, The Weizmann Institute, Rehovot, Israel) and Dr. Christopher Walsh (Howard Hughes Medical Institute, Beth Israel Deaconess Medical Center, Boston, MA). Comparable results were obtained with all three antibodies against DCX.

Imaging and analysis. Imaging of fixed cells was performed with a Carl Zeiss Axiovert 200M to which was interfaced a Cooke sensicam cooled CCD camera. The camera, shutter, and motorized microscope components were controlled using iVision software (Biovision Technologies). Filter sets optimized for multiple labeling analyses using cyanine 2 and 5 and Rhodamine Red-X were obtained from Chroma Technology. Images were acquired using the full usable area of the CCD chip, which measured $1376 \times 1024$ pixels and stored in full 12-bit format for processing and analysis. To reveal the general morphology of neurons and to quantify total axonal length, fluorescent images were obtained with a $25 \times / 0.8$ numerical aperture (NA) or $40 \times / 1.3$ NA Plan-Neofluar oil objective. To avoid selection bias in these analyses, we predetermined a minimum number of axons to analyze, usually 20-40, and took images of every neuron encountered up to the predetermined number. Axonal length was measured with the length-measuring tool in iVision. High-resolution images of axons and growth cones were obtained with the $100 \times / 1.3 \mathrm{NA}$ Plan-Neofluar oil objective. Image processing was performed with various tools in iVision; ratio imaging and the segmented mask procedure, which measures fluorescence intensity in user-defined segments along the axon and in the growth cone, were performed with customized routines developed in Oncor Imaging Software (Brown et al., 1992). Images were imported into Adobe Photoshop (Adobe Systems) to prepare figures for presentation (version CS3 extended); unless otherwise indicated, linear methods were used to enhance image brightness and contrast for presentation.

Time-lapse imaging was performed to determine the relationship of focal axonal accumulations of DCX described here with "growth cone like waves" described by Ruthel and Banker (1998). These latter structures consist of prominent lamellipodia that move within the axon at rates of $\sim 3 \mu \mathrm{m} / \mathrm{min}$. Neurons were grown on gridded coverslips. Axons with discrete lamellipodial structures along their length were identified and imaged using time-lapse phase microscopy for $10 \mathrm{~min}$ intervals. The neurons were then fixed and stained for actin and DCX as described 

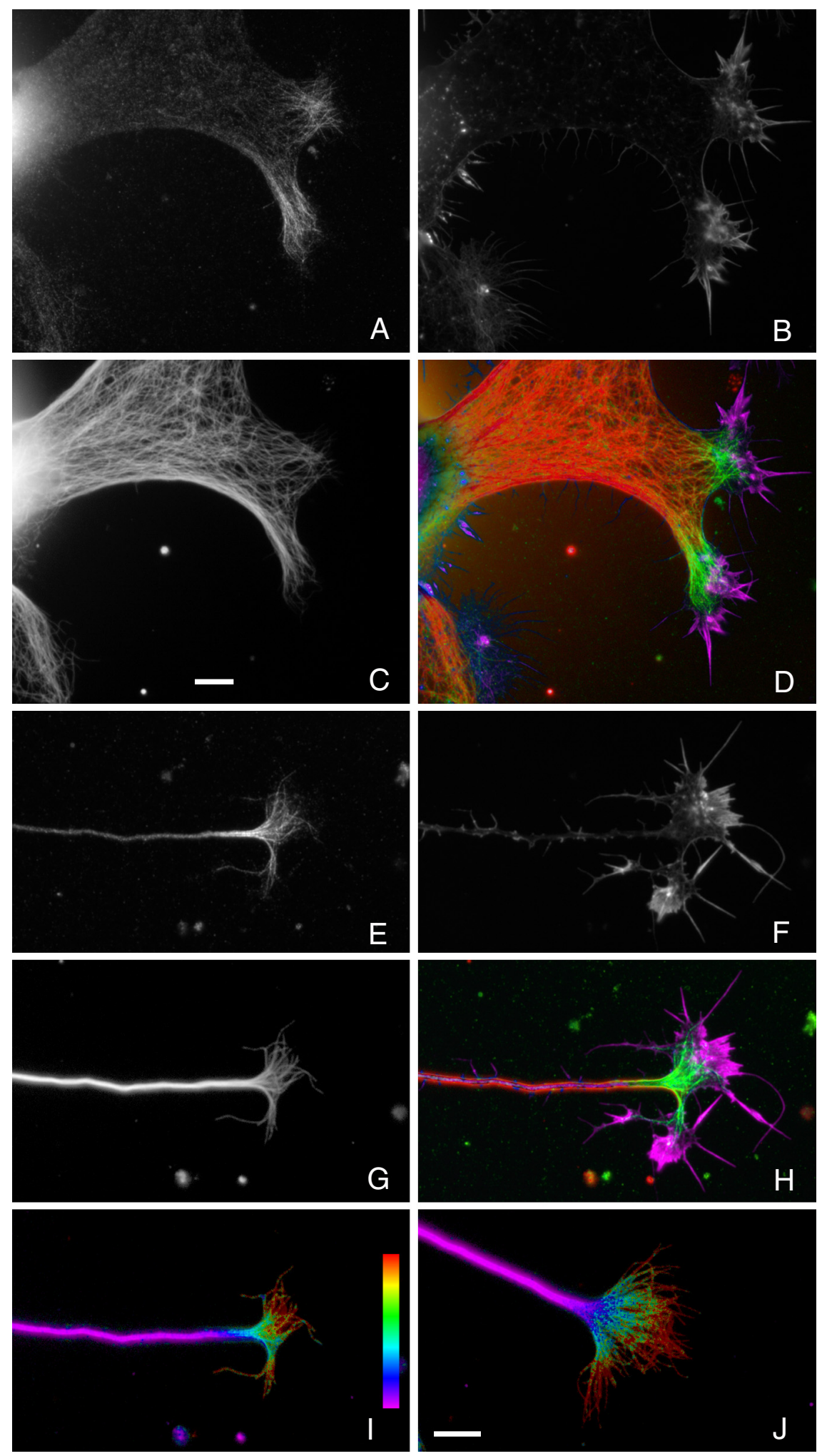

Figure 1. $D C X$ is associated with microtubules specifically in regions that stain prominently for actin filament assemblies indicative of protrusive activity. Sympathetic neurons were fixed with glutaraldehyde in the presence of nonionic detergent to preserve both microtubules and actin filaments and to remove unassembled pools of tubulin and actin. $\boldsymbol{A}-\boldsymbol{C}$ show a large lamella extending from a neuron stained for DCX, actin filaments, and microtubules, respectively. $\boldsymbol{D}$ shows a color overlay of these images with microtubules in red, DCX in green, and actin filaments in violet. In such lamellae, which represent very early stages in axonal formation (Yu et al., 2001), DCX is concentrated specifically on the distal parts of microtubules that extend into regions of strong actin staining that are probably nascent growth cones. $\boldsymbol{E}-\boldsymbol{G}$ show high-magnification views of a growth cone stained for $D C X$, actin filaments, and microtubules, respectively. Hshows a color overlay of these images with microtubules in red, DCX in green, and actin filaments in violet. DCX is concentrated on microtubules throughout the growth cone, including the portions that extend into its above. To directly establish the relationship between the focal accumulations of DCX and the growth-cone-like waves, green fluorescent protein (GFP)-DCX was expressed in neurons. The GFP-DCX construct was generously provided by Dr. Orly Reiner. The construct was introduced into dissociated neurons using the Amaxa nucleofection device as described previously (Hasaka et al., 2004; He et al., 2005; Myers et al., 2006). Focal accumulations were identified in living neurons expressing GFPDCX. A fluorescent image was obtained of the cell, followed by a time-lapse sequence using phase optics (10 min duration), followed by another fluorescence image. Time-lapse imaging used the $40 \times / 1$.3 NA Plan-Neofluar oil objective; images were acquired at $2 \mathrm{~s}$ intervals using an exposure time of $0.1 \mathrm{~s}$.

\section{Results}

We used dissociated cultures of sympathetic and hippocampal neurons to explore DCX functions in relation to axonal morphogenesis. These two culture systems exhibit distinctive growth behaviors. Sympathetic neurons from the superior cervical ganglia are PNS neurons that are specialized to extend long axons very rapidly, at rates of $\sim 30 \mu \mathrm{m} / \mathrm{h}$ or more. The axons also branch extensively, almost exclusively via the process of growth cone bifurcation. Furthermore, by manipulating the substrate and media composition, the initiation of axonal growth can be triggered at the experimenter's discretion (Rivas et al., 1992; Slaughter et al., 1997; Yu et al., 2001), facilitating the examination of DCX localization at very early steps in axonal formation. Hippocampal neurons are CNS neurons that undergo a fairly stereotyped sequence of developmental events in culture that results in the generation of axonal and dendritic polarity (Dotti et al., 1988). In the course of these events, the axon extends at a modest rate by comparison with sympathetic neurons, and axons branch principally by forming collaterals. In the present studies, we have localized DCX in growing axons of cultured sympathetic and hippocampal neurons and determined the effects of DCX depletion on axonal morphology of these neurons.

transition zone and peripheral domains. Ratio images depicting the abundance of $D C X$ relative to microtubules in this and another growth cone are shown in $I$ and $J$, respectively. These ratio images clearly show that the abundance of DCX on microtubules increases in a proximal-to-distal manner from the base of the growth cone toward the peripheral domain, reaching the highest concentration on the splayed microtubules in the peripheral domain. Scale bars, $7.2 \mu \mathrm{m}$ (Capplies to $\boldsymbol{A}-\boldsymbol{D}, \boldsymbol{J}$ applies to $\boldsymbol{E}-\boldsymbol{J})$. 


\section{DCX is preferentially enriched on} microtubules in regions containing prominent actin filament assemblies Initial staining analyses using fixation with cold methanol or glutaraldehyde revealed prominent staining for DCX in the cell body and growth cone, with little or no staining apparent along the axonal shaft (Fig. 1 and data not shown). Comparable results were obtained with the three different DCX antibodies used in the present studies. These results confirm previously published results of DCX localization in cultured neurons (Friocourt et al., 2003; Gdalyahu et al., 2004; Schaar et al., 2004).

More detailed inspection reveals several additional features of DCX localization in growth cones of cultured sympathetic neurons. First, DCX clearly and unambiguously colocalizes with microtubules (Fig. 1). Furthermore, within the growth cone, DCX was only detected in close association with microtubules. We did not detect any background of diffuse DCX staining that exceeded that of controls using nonimmune antibodies in place of DCX antibodies, nor did we detect any concentrated foci of DCX not colocalized with microtubules. This was true for cells fixed with cold methanol, which should precipitate all DCX in the growth cone and the combined fixation/ extraction procedure that is expected to remove unassembled DCX. These results are based on visual inspection of DCX staining of many growth cones using three different DCX antibodies and various enhancing procedures to accentuate DCX staining. Second, most or all of the microtubules in growth cones at the tips of long, well developed axons appear to be decorated with DCX (Fig. $1 E, G$ ). Furthermore, visual inspection suggests that the relative abundance of DCX on the microtubules increases in a proximal-to-distal manner within the growth cone, an impression confirmed by ratio imaging (Fig. 1I,J). Growth cones consist of a central domain (rich in microtubules and contiguous with the neck region), a peripheral domain (the distal actin-rich region, which includes the lamellae and filopodia of the growth cone), and a transition zone between them. Thus, within growth cones, the microtubule polymer at the axongrowth cone neck region has the lowest relative abundance of DCX, whereas the polymer extending into the transition zone and peripheral domain of the growth cone has the highest relative levels of DCX.

Cultured sympathetic neurons initiate axonal growth by extending large lamellae that elongate to become the nascent axon. These nascent axons have a large diameter and are tipped by large growth cones (Slaughter et al., 1997; Yu et al., 2001). Figure 1A-D shows a large lamella characteristic of very early axon initiation. Although microtubules fill these structures, DCX is highly concentrated only on the portions of the microtubules that extend into the distal reaches of select regions of the lamella, whereas
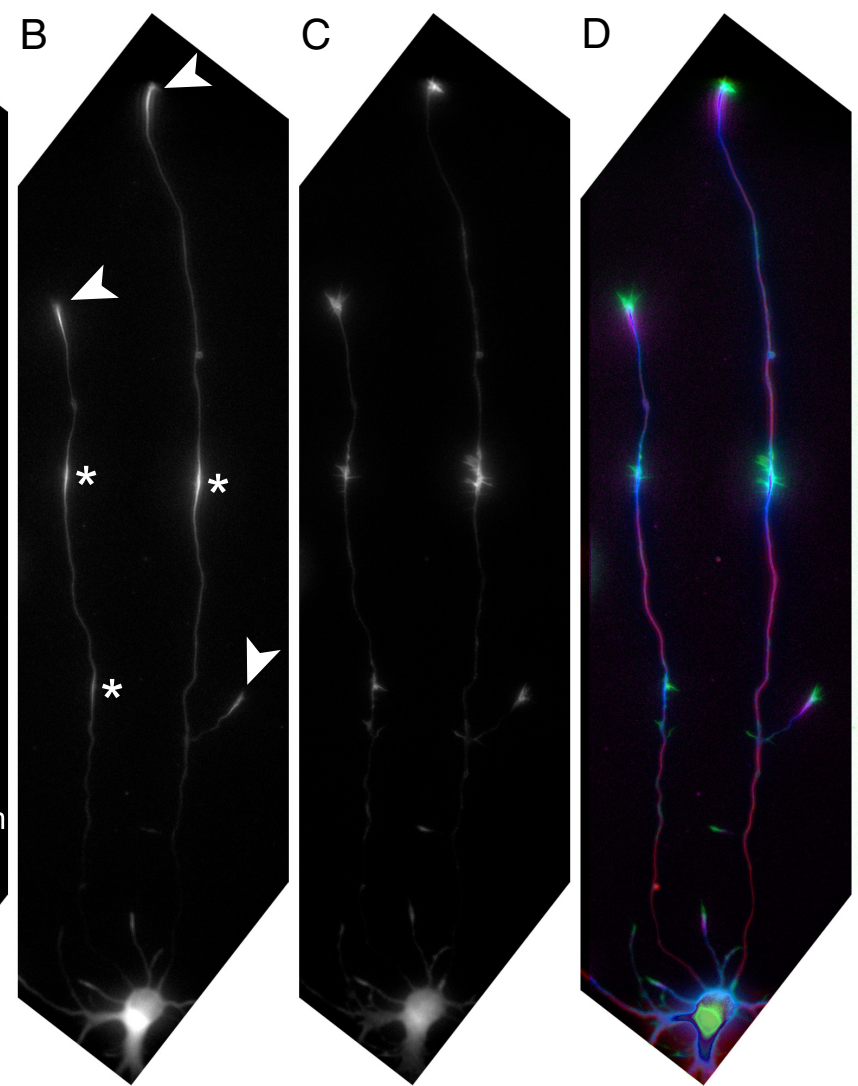

Figure 2. DCX in cultured hippocampal neurons is concentrated in the growth cone and at discrete sites along the axon wo-day-old cultures of hippocampal neurons were fixed extracted with glutaraldehyde and then triple stained to reveal micro-

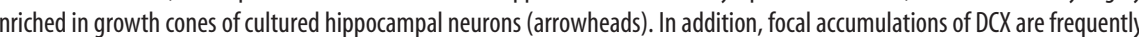
pultiple examples of the spatial association of DCX with regions staining strongly for actin filaments within the confines of a relatively small figure. Quantitative analyses of staining intensity of microtubules, DCX, and actin filaments along the shorter axon are shown in Figure 4 . The small arrow in $\boldsymbol{A}$ shows a varicosity. Scale bar, $29 \mu \mathrm{m}$.

comparatively little DCX is present on microtubules located more proximally in these structures. The regions of the lamellae in which the microtubules are rich in DCX also contain prominent actin-based structures indicative of active protrusive activity. Collectively, these findings indicate the following: within the axons and growth cones of cultured sympathetic neurons, DCX is highly enriched in the growth cone, with little to none detectable in the axonal shaft; within the growth cone, DCX staining was only observed in association with microtubules; and the microtubule polymer richest in DCX was localized to the transition zone and peripheral domain of the growth cone, regions that also display actin-rich structures indicative of vigorous protrusive activity.

The localization of DCX in cultured hippocampal neurons shows both similarities and differences with respect to that seen in cultured sympathetic neurons. The principal similarity is that DCX is usually very abundant in growth cones, in which it associates with microtubules (Figs. 2, 3). Furthermore, the relative abundance of DCX on microtubules increases progressively from the axon-growth cone junction to the peripheral domain of the growth cone (Fig. 3E) (see also Fig. 6D) and the polymer richest 

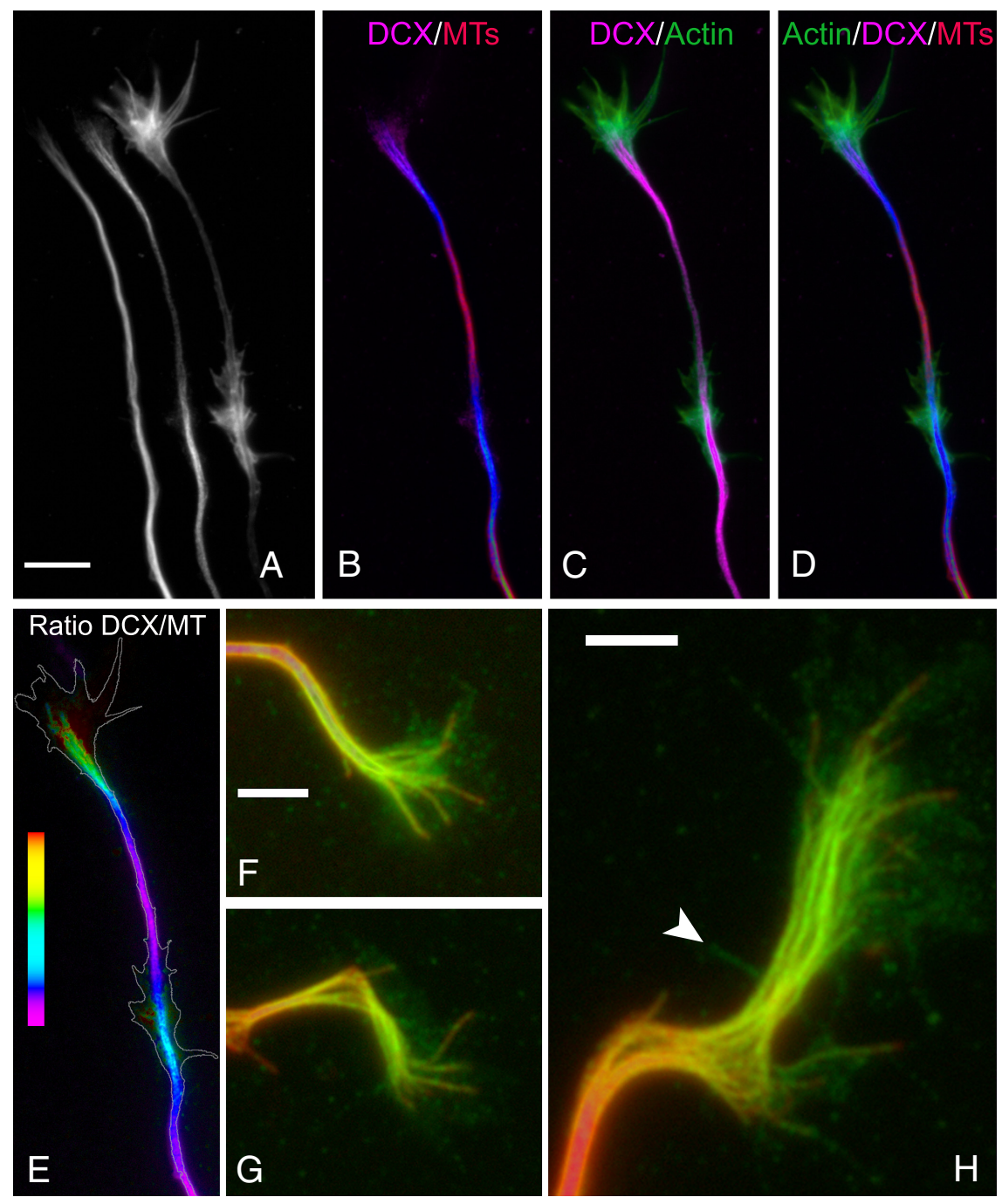

Figure 3. Higher-resolution analyses of microtubules, $D C X$, and actin staining in growth cones and focal axonal accumulations of DCX. The figure shows the distal axon and growth cone of cultured hippocampal neurons. $A$ shows, from left to right, images of microtubule (MT), DCX, and actin filament staining. $\boldsymbol{B}-\boldsymbol{D}$ show overlay images as indicated, with microtubule staining in red, $D C X$ staining in violet, and actin staining in green. DCX staining is strong in the growth cone and at a site along the axon a bit proximal to the growth cone (these accumulations appear blue in the $D C X /$ microtubule overlay image). Within the growth cone, $D C X$ staining primarily colocalizes with microtubules, and the polymer rich in DCX extends into the actin-rich region of the growth cone. Note also that the region of the axon with the focal accumulation of $D C X$ is also rich in actin filaments that form prominent lamellipodia. $\boldsymbol{E}$ shows a ratio image depicting the abundance of $D C X$ relative to microtubules; the white outline represents the contour of actin staining. In the growth cone, the ratio increases progressively from the axon-growth cone neck region to the distal extent of the microtubule array (for a ratio image of another neuron, see Fig. 6). The ratio of $D C X$ staining to microtubule staining is also elevated within the focal accumulation of DCX along the axon. Scale bar (in $\boldsymbol{A}$ ): $\boldsymbol{A}-\boldsymbol{E}, 7.2 \mu \mathrm{m}$. $\boldsymbol{F}-\boldsymbol{G}$ show zoomed images of $D C X$ (in green) and microtubule (in red) staining in three different growth cones. The colocalization of DCX with microtubules is readily apparent. There is also glow in the DCX channel that is not microtubule associated. Note that nonlinear enhancing methods were used to accentuate this glow without saturating that associated with microtubules. See Results for discussion of the possible significance of this non-microtubule fluorescence. The arrowhead in $\boldsymbol{H}$ identifies a filopodia detected by phalloidin staining (data not shown). Scale bars, $3.2 \mu \mathrm{m}$.

in DCX localizes to peripheral growth cone regions that also stain strongly for actin filaments. Visual inspection revealed that, although most growth cones stained strongly for DCX, some stained poorly. It was also our impression that the growth cones that stained strongly for DCX also stained strongly for actin filaments and, conversely, that growth cones that stained poorly for DCX also stained poorly for actin (data not shown). To examine these relationships in more detail, we evaluated all growth cones for the presence or absence of an abrupt increase in staining for DCX and actin relative to the axonal shaft immediately

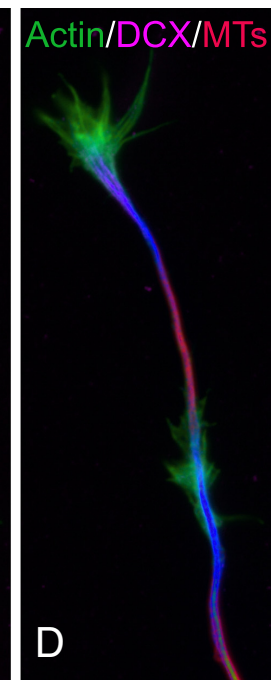

behind the growth cone. Of $>700$ growth cones evaluated, $73 \%$ showed an obvious accumulation of both actin and DCX, whereas in $26 \%$, staining for both actin and DCX was dim and did not increase above that in the axonal shaft immediately proximal to the growth cone. Thus, for $99 \%$ of the growth cones examined, relatively strong staining for DCX predicted relatively strong staining for actin filaments and vice versa, whereas relatively weak staining for actin filaments predicted weak staining for DCX. Based on morphological considerations, it is our impression that growth cones that stained strongly for both actin and DCX had been actively extending, whereas those that stained weakly for actin and DCX had not been growing or had been retracting at the time of fixation.

Two differences were observed in the localization of DCX in hippocampal neurons compared with sympathetic neurons. First, we did note fluorescence in the DCX channel in growth cones that was not associated with microtubules (Fig. $3 F-H)$. These images were processed using nonlinear methods to selectively enhance the brightness of this fluorescence relative to that associated with microtubules. Occasionally, DCX fluorescence was observed in filopodia (Fig $3 H$, arrowhead). However, more typically, it appeared diffuse or punctate within the body of the growth cone. This may represent unassembled DCX that was not removed during the combined fix/extract procedure and/or DCX associated with components of the growth cone other than microtubules. It is also possible that some or all of this fluorescence is nonspecific background. In this regard, cells stained with a non-immune antibody instead of the DCX antibody exhibited similar non-microtubule fluorescence, although it is our impression that the intensity was less than with the DCX antibody. Additional information is required to more fully evaluate the significance of this non-microtubule fluorescence.

The other difference in DCX staining was observed in the axonal shaft. Whereas the shafts of cultured sympathetic axons exhibited little staining for DCX, we commonly observed one or more discrete sites of relatively prominent DCX staining along the shafts of axons of cultured hippocampal neurons (Figs. 2, *; 3 ). These were especially common in axons $\geq 100 \mu \mathrm{m}$ in length but were also encountered in shorter axons. These sites appeared as very abrupt focal increases in DCX staining. The focal accumulations of DCX were of variable length, ranging from a few micrometers for the smaller accumulations to $\sim 12-15 \mu \mathrm{m}$ for the longer accumulations. This point can be seen by inspection in Figures 2 and 3 and is also demonstrated quantitatively in Figure 


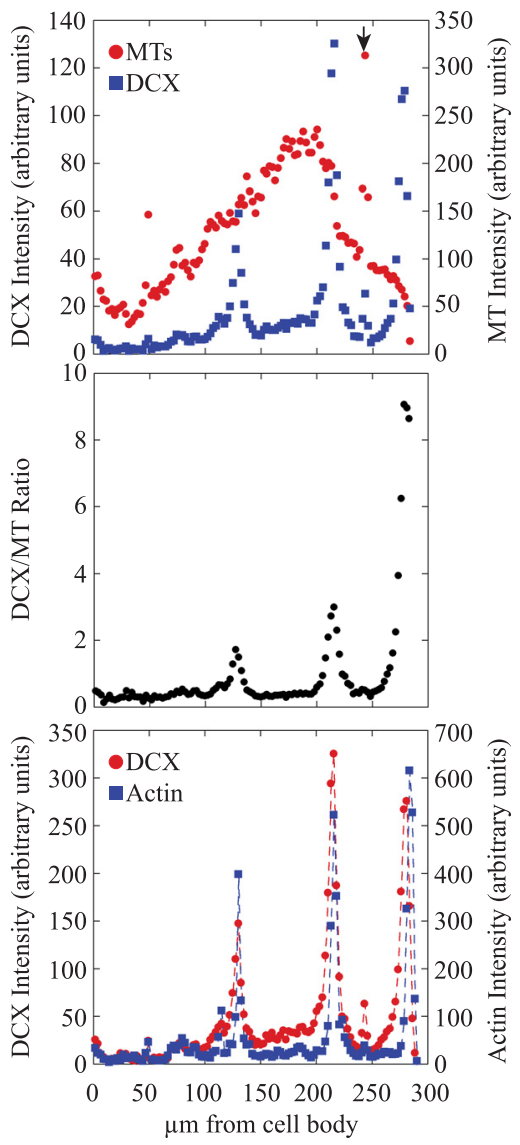

Figure 4. Focal accumulations of $D C X$ along axons coincide with focal accumulations of actin filaments. Fluorescence intensity (in arbitrary units) attributable to microtubules (MT), DCX, and actin filament staining was quantified in consecutive $2.5 \mu \mathrm{m}$ segments of the shorter axon of the neuron depicted in Figure 2. The top shows fluorescence intensity for microtubules and $D C X$ along the length of the axon and in the growth cone. Relatively strong $D C X$ staining is seen at two discrete sites along the axon and in the growth cone. The arrow in the top identifies the location of an axonal varicosity that stains very strongly for microtubules but comparatively weakly for DCX (see Fig. 2). The middle shows the ratio of DCX-to-microtubule staining intensity along the axon. Note that the ratio spikes sharply at the axonal sites of focal accumulations of $D C X$ and in the growth cone. The bottom shows fluorescence intensity for actin filaments and $D C X$ along the axon and in the growth cone. Note the strong coincidence between the locations of focal accumulations of $D C X$ and strong staining for actin filaments. It appears that the focal accumulations of actin filaments are concentrated at the more distal regions of the focal $D C X$ accumulations, a view that is also apparent by visual inspection of higher-magnification views of DCX and actin filament staining (see Fig. 3).

4. Also, the focal accumulations of DCX were associated with focal accumulations of actin filaments (Figs. 2-4). These accumulations of actin filaments were variable in appearance but could be divided into two general categories based on the presence of lamellipodia. Approximately $40 \%$ of the focal actin filament accumulations (of 398 examined) had prominent lamellipodia extending from the sides of the axon, and these often had filopodia as well. The remaining $\sim 60 \%$ of the accumulations lacked obvious lamellipodia, although filopodia were often present (supplemental Fig. 1, available at www.jneurosci.org as supplemental material). As described below, the focal accumulations of DCX and actin that have obvious lamellipodia are highly dynamic, undergoing a time-dependent proximal-to-distal redistribution, and their lamellar appearance changes constantly as they advance within the axon.

We quantified the extent to which focal accumulations of DCX coincided with focal accumulations of actin filaments. For these analyses, we only evaluated neurons that were completely isolated from neighboring neurons to minimize the potential for axonal fasciculation confounding the results. Of 379 axonal accumulations of DCX evaluated, 377 (>99\%) were associated with focal accumulations of actin filaments. Similarly, $95 \%$ of the 398 focal accumulations of actin filaments evaluated were associated with focal accumulations of DCX.

More detailed inspection of the focal accumulations of DCX and actin along the axon revealed that they do not overlap perfectly. For most, the length of the focal accumulation of DCX exceeded that for actin filaments, and the actin filament accumulation was displaced toward the distal end of the DCX accumulation (Figs. 2-4). Also, the accumulation of DCX mostly colocalizes with the microtubule array of the axonal shaft, with relatively little DCX staining extending into the actin-rich filopodia and lamellipodia. However, in most focal accumulations, individual or small bundles of microtubules could be seen extending from the axonal shaft into the actin-rich accumulations, and these were decorated with DCX (Fig. 5). It is also our impression that a direct relationship exists between the length and intensity of the focal accumulations of DCX and the length and intensity of the actin filament accumulations. For example, in Figure 2, the three focal accumulations of DCX apparent in the axons $\left(^{*}\right)$ differ in staining intensity and also in length, and a corresponding difference also exists in the intensity and length of their associated actin filament accumulations, and this can be seen in the quantitative analyses of Figure 4 as well.

Finally, focal accumulations of both actin and DCX were observed in the proximal, middle, and distal portions of the axon. Generally, they were not located at sites in which well developed branches emerged from the axonal shaft. However, as discussed below, they do appear to be associated with the formation of collateral branches.

\section{Microtubule domains rich in DCX are deficient in tau}

DCX is only one of several microtubule-associated proteins (MAPs) present in axons. Another axonal MAP is tau, which is present on microtubules all along the axon and in the growth cone (Black et al., 1996; Kempf et al., 1996; Mandell and Banker, 1996). Comparison of DCX and tau association with growth cone microtubules revealed that the portions of growth cone microtubules especially rich in DCX are deficient in tau, whereas the portions that stain well for tau are deficient in DCX, and this is true for both cultured hippocampal (Fig. 6) and sympathetic (supplemental Fig. 3, available at www.jneurosci.org as supplemental material) neurons. Ratio imaging makes this point particularly clearly by showing that the tau-to-microtubule ratio declines in a proximal-to-distal direction within the growth cone as the DCX-to-microtubule ratio increases (Fig. 6) (supplemental Fig. 3, available at www.jneurosci.org as supplemental material). The same point is also made by analyses using the segmented mask procedure (Fig. 7), which show that the tau-tomicrotubule ratio peaks a bit proximal to the growth cone and declines steadily from there to the distal extent of the microtubule array, whereas the DCX-to-microtubule ratio begins to increase near the axon-growth cone junction and increases steadily to the end of the microtubule array (supplemental Fig. 2, available at www.jneurosci.org as supplemental material). The poor tau staining on the very distal portions of growth cone microtubules is not secondary to shielding or competition by the antibodies used to visualize DCX because tau staining on these microtubules also appeared very dim in control experiments in which a nonimmune antibody was used in place of the DCX antibody (Fig. 

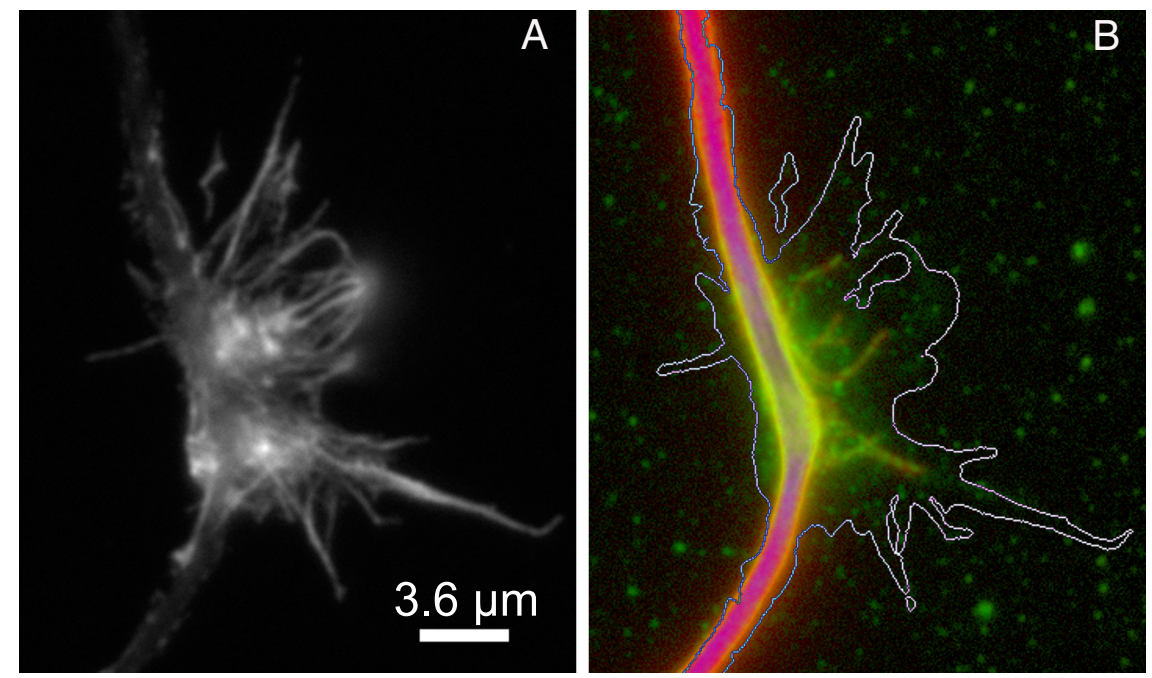

Figure 5. Splayed microtubules at sites of focal accumulations of $D C X$ along the axon. $A$ shows actin filament staining associated with a focal accumulation of DCX. B shows an overlay of DCX (green) and microtubule (red) staining in the same region; the white line is an approximate outline of the actin staining. Note that several microtubules splay out from the main microtubule bundle and that these are decorated with DCX. Nonlinear methods were used to enhance the staining associated with the splayed microtubules without grossly saturating the staining associate with the microtubule bundle of the axonal shaft. Scale bar, $3.6 \mu \mathrm{m}$.
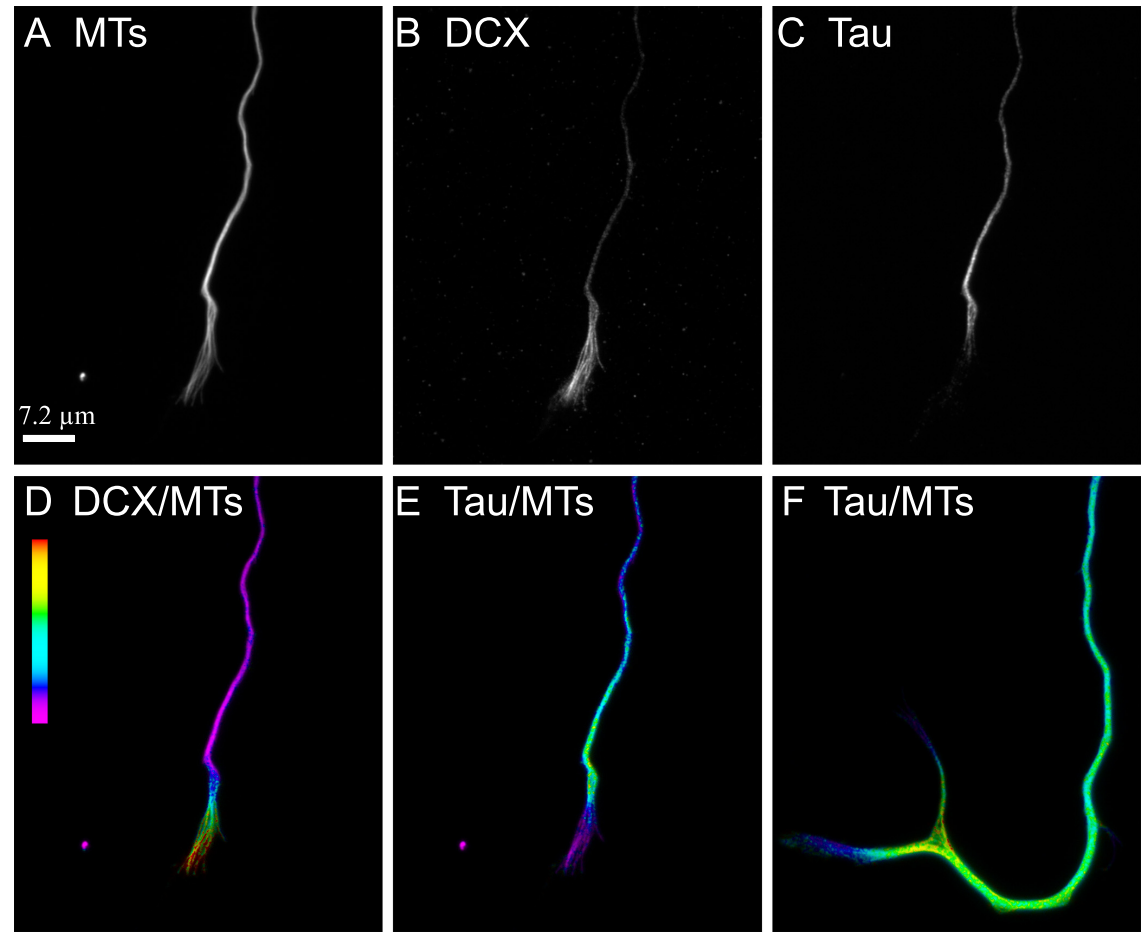

Figure 6. Tau and $D C X$ have complementary distributions on growth cone microtubules. In these analyses, hippocampal neurons were incubated with the tau antibody first, followed by that for DCX and then for tubulin. The cells were then incubated with a mixture of the requisite secondary antibodies. $A-C$ show microtubule (MT), DCX, and tau staining in the distal axon and growth cone. $\boldsymbol{D}$ and $\boldsymbol{E}$ show ratio images depicting the relative abundance of $D C X$ to microtubules or tau to microtubules, respectively. The ratio of DCX-to-microtubules increases progressively from the axon-growth cone neck region to the distal end of the microtubule array in the growth cone, whereas the exact converse is true for tau. $\boldsymbol{F}$ shows a ratio image of a control neuron triple stained for microtubules, tau, and a non-immune antibody in place of the $D C X$ antibody. Even in the absence of DCX antibodies, the ratio of tau-to-microtubules declines in a proximal-to-distal manner in the growth cone. Thus, the proximodistal decline of tau staining of growth cone microtubules is not a result of exclusion/competition attributable to binding of $\mathrm{DCX}$ antibodies.

$6 F$ ) or in neurons just double stained for tubulin and tau (Black et al., 1996). Tau staining is also diminished in the focal accumulations of DCX seen along axons of cultured hippocampal neurons (Fig. 7).

\section{Experimental actin depolymerization} disrupts DCX localization

The observations described above indicate that the distribution of DCX is tightly regulated in growth cones and along the axonal shaft such that it is preferentially concentrated on microtubules in regions rich in actin filaments. An obvious issue that arises from these observations is whether the localization of DCX is in some way dependent on the actin system. To address this issue, we used latrunculin to determine whether the localization of DCX is altered by acute depolymerization of actin filaments. Based on previous studies showing that treatment of cultured hippocampal neurons with high doses of latrunculin very effectively depolymerizes actin filaments over a time course of several minutes (Sankaranarayanan et al., 2003, Roy et al., 2008), we found that treatment with $25 \mu \mathrm{M}$ latrunculin for times as short as $3 \mathrm{~min}$ reduced phalloidin staining to negligible amounts (Fig. 8). Time-lapse imaging revealed that such latrunculin treatments caused a rapid collapse of the growth cone but little axonal retraction after 3 or $10 \mathrm{~min}$ of treatment (data not shown).

Representative low-magnification images of control and latrunculin-treated neurons are shown in Figure 8. Control neurons exhibit strong staining for both actin and DCX in the growth cone and at focal sites along the axon. At this magnification, there were no detectable actin structures in the axon or growth cone after 3 or $10 \mathrm{~min}$ of latrunculin treatment. However, at higher magnification, many neurons exhibited occasional actin containing structures along the axon and its tip (data not shown). These typically took the form of discrete punctate or dash-like structures. The pattern of DCX staining changed in response to latrunculin treatment. As early as 3 min of treatment, the frequency of focal accumulations of DCX along the axon was dramatically reduced, and this reduction was even more pronounced by $10 \mathrm{~min}$ of drug treatment (Fig. 9A). The mean number of focal accumulations of DCX per axon was $0.78 \pm$ 0.71 (mean $\pm \mathrm{SD} ; n=50$ axons) in control axons versus $0.20 \pm 0.40(n=50)$ and $0.10 \pm 0.30(n=50)$ in axons treated with latrunculin for 3 or $10 \mathrm{~min}$, respectively; the differences between control and drugtreated axons are statistically highly significant (Student's $t$ test, $p<0.0001$ ). The enrichment of DCX in growth cones also changed as a result of latrunculin treatment but with a different time course (Figs. 8, $9 B)$. Specifically, DCX was enriched in 92 and $82 \%$ of the growth cones of control neurons $(n=50)$ and neurons treated with 
latrunculin for $3 \min (n=50)$, respectively. However, by $10 \mathrm{~min}$ of drug treatment, only $22 \%(n=50)$ of the growth cones were enriched in DCX, and this decrease was highly significant $\left(\chi^{2}\right.$ test, $p<$ $0.0001)$. After $10 \mathrm{~min}$ of drug treatment, most axons exhibited relatively uniform staining for DCX (Fig. 8), and it was our impression that the overall intensity of DCX staining along the axon was greater than the low baseline seen along the length of control axons.

At higher magnification, the focal accumulations that remained after latrunculin treatment resembled those in control axons in terms of DCX staining (data not shown). In terms of phalloidin staining, very little remained in association with focal accumulations of DCX, and these axonal regions were not notably different from neighboring regions without accumulations of DCX in terms of the presence of occasional phalloidin staining structures. Control growth cones typically were enriched in DCX, much of which localized with microtubules. As described above, DCX was enriched in the tips of axons treated with latrunculin for $3 \mathrm{~min}$ but not for $10 \mathrm{~min}$. Because the tips of latrunculin-treated axons generally were not well spread, it was difficult to determine whether the DCX therein was microtubule associated. In particularly favorable preparations, it was our sense that some of the remaining DCX was microtubule associated, but some also appeared in regions without appreciable microtubule staining (data not shown).

\section{The focal accumulations of DCX in axons undergo slow proximal-to-distal movement within the axon}

The focal accumulations of DCX and actin filaments described here bear a striking resemblance to structures originally described by Ruthel and Banker (1998), which moved anterogradely at $\sim 3$ $\mu \mathrm{m} / \mathrm{min}$ in axons of cultured hippocampal neurons (Flynn et al., 2009). These contained abundant actin filaments relative to adjacent axonal regions and also exhibited prominent filopodial and lamellipodial extensions. These authors referred to these as "growth cone like waves"- growth cone like because they exhibited protrusive activity similar to that of the growth cone and also were enriched in actin and GAP43, proteins typically enriched in growth cones, and waves because of their proximal-to-distal movement. We determined whether the focal accumulations of DCX described here correspond to the "growth cone like waves" described by Ruthel and Banker. First, we performed time-lapse phase imaging of focal protrusive structures along the axon that consisted of obvious lamellipodia and then fixed the imaged cells and stained them for DCX and actin filaments. Of 27 lamellar structures imaged in this manner, 12 underwent a proximal-todistal redistribution at an average rate of $2.1 \pm 0.89 \mu \mathrm{m} / \mathrm{min}$ (mean $\pm \mathrm{SD}$; minimum, $1.55 \mu \mathrm{m} / \mathrm{min}$; maximum, $3.95 \mu \mathrm{m} /$ min), and all of these stained relatively brightly for DCX and actin filaments (data not shown). Of the 15 lamellar structures that did not move during the time-lapse sequence, only six stained well for both DCX and actin filaments, four others stained well for actin but not for DCX, and the remaining five stained weakly for both actin filaments and DCX. We note that lamellar structures were only observed for $10 \mathrm{~min}$ in these time-lapse imaging experiments. Although this time is more than sufficient to reveal persistent movement at rates $\geq 1 \mu \mathrm{m} / \mathrm{min}$, the time may be too short to unambiguously reveal lamellar structures that move appreciably more slowly than this or that undergo relatively prolonged pauses in their movement. Thus, the frequency with which lamellar structures move may be higher than indicated in these analyses. Collectively, these observations suggest that the majority of the moving protrusive structures correspond to the focal accumulations of DCX described above. To directly evaluate whether the focal accumulations of DCX undergo a proximal-to-distal movement together with these protrusive structures, we performed time-lapse imaging on neurons expressing GFP-DCX.

The distribution of GFP-DCX along the axons of expressing neurons varied apparently in relation to the degree of expression. In many neurons, GFP-DCX fluorescence was quite bright and was present all along the axon and in the growth cone, and there was little or no indication of focal accumulations along the axon. In some of these cells, a notable increase in growth cone fluorescence was apparent, whereas in others, growth cone fluorescence was bright but not appreciably enhanced relative to that of the axon behind it. In a minority of neurons, GFP-DCX fluorescence was relatively dim, and in these cells the pattern of fluorescence resembled that of neurons stained for DCX. Specifically, GFPDCX fluorescence increased dramatically from the distal axon into the growth cone, and a portion of the cells also exhibited very clear focal accumulations of DCX (Fig. 10). The present analyses focused specifically on neurons with this latter appearance.

For cells exhibiting obvious focal accumulations of GFP-DCX along the axon, three images were obtained in the following se- 

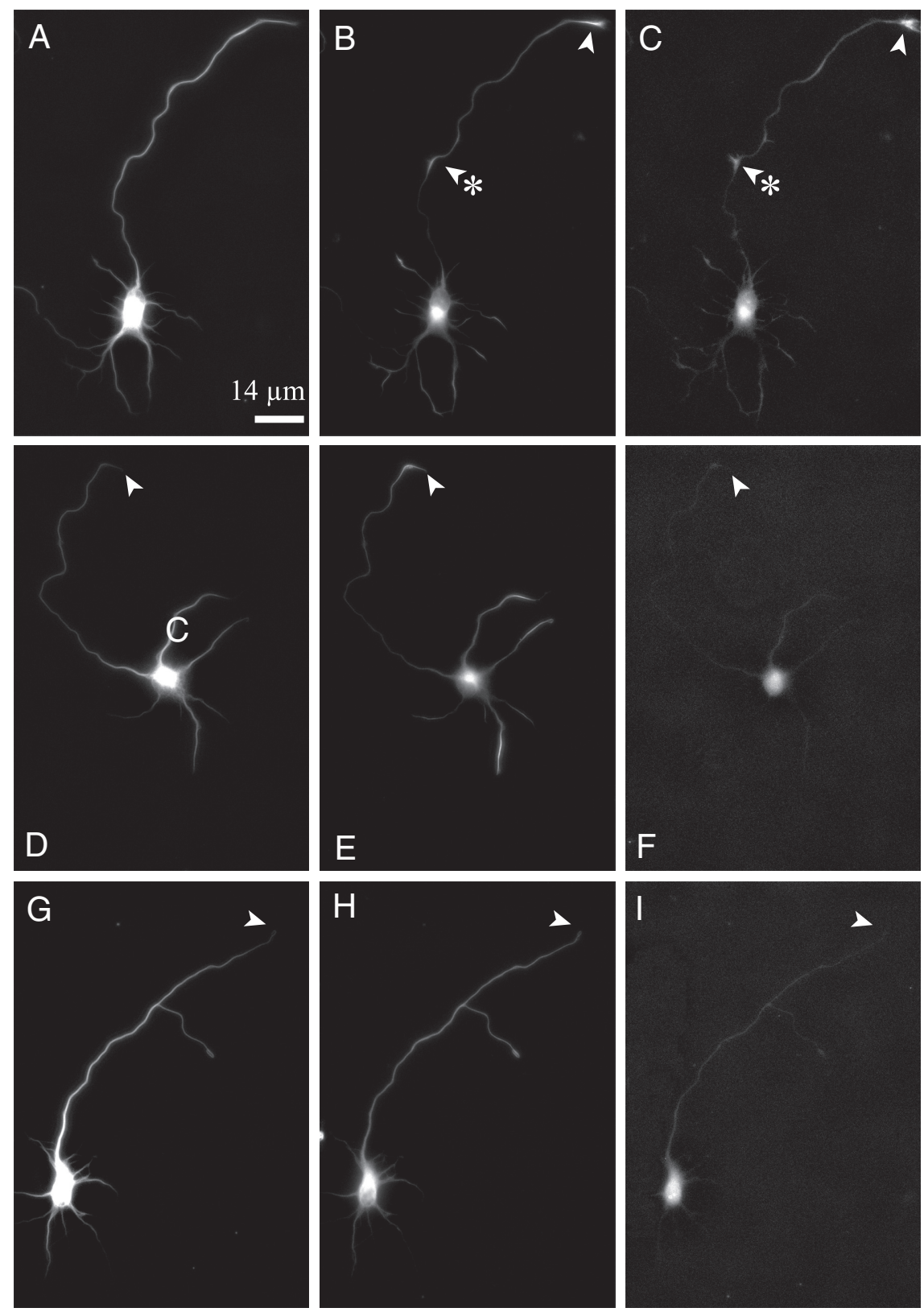

Figure 8. Acute actin depolymerization induced by treatment with latrunculin leads to a loss of focal accumulations of $D C X$ along the axon and in the growth cone. Shown are images of microtubule $(\boldsymbol{A}, \boldsymbol{D}, \boldsymbol{G}), \mathrm{DCX}(\boldsymbol{B}, \boldsymbol{E}, \boldsymbol{H})$, and phalloidin $(\boldsymbol{C}, \boldsymbol{F}, \boldsymbol{I})$ staining of a control neuron $(\boldsymbol{A}-\boldsymbol{C})$ and neurons treated with $25 \mu \mathrm{m}$ latrunculin for $3 \mathrm{~min}(\boldsymbol{D}-\boldsymbol{F})$ or $10 \mathrm{~min}(\boldsymbol{G}-\boldsymbol{I})$. Arrowheads identify axon tips; arrowheads with asterisks $(\boldsymbol{B}, \boldsymbol{C})$ identify a focal accumulation of $D C X$ and actin filaments. Scale bar, $14 \mu \mathrm{m}$.

ing anterogradely at an average rate of $1.57 \pm 1.45 \mu \mathrm{m} / \mathrm{min}$ (mean $\pm \mathrm{SD}$; minimum, $0.34 \mu \mathrm{m} / \mathrm{min}$; maximum, $7.47 \mu \mathrm{m} /$ $\mathrm{min})$. Data from one such cell are shown in Figure 10 and supplemental Movie 1 (available at www.jneurosci.org as supplemental material). The one focal accumulation that moved retrogradely moved at $2.19 \mu \mathrm{m} / \mathrm{min}$. All of the focal accumulations of GFP-DCX that moved were associated with lamellipodia that exhibited very active protrusive activity, and the focal accumulation of GFP-DCX appeared to advance in concert with the advancing lamella. Retrospective analyses suggested that focal accumulations of GFP-DCX associated with spread regions that exhibited very active lamellar and/or filopodial activity were likely to advance the axon during the subsequent $10 \mathrm{~min}$ period, whereas axonal regions enriched in GFPDCX but that were relatively quiescent in terms of lamellar activity were unlikely to move during the subsequent $10 \mathrm{~min}$.

\section{Experimental studies of DCX function}

To explore functions of DCX, we used DCX-specific siRNA to knock down DCX in cultured sympathetic and hippocampal neurons. For these experiments, freshly dissociated neurons were transfected with control or DCX siRNA and then cultured for $3 \mathrm{~d}$, which was sufficient to significantly reduce DCX levels in many of the neurons. The control and DCX-depleted neurons were then dissociated and replated under conditions that permitted vigorous regrowth of axons (He et al., 2005; Myers et al., 2006) and examined at 1-2 d after replating. With this culture regimen, neurons are challenged to generate axons anew after depletion of DCX. In the absence of any manipulations other than replating, the cells survive well and grow vigorously, recapitulating the normal developmental sequence, although as shown below, a bit faster than freshly plated cells. In other studies using this culture regimen, various manipulations have

quence: first a fluorescence image, then a 10 min time-lapse sequence using phase microscopy, and finally another fluorescence image. The fluorescence images would reveal the location of the focal accumulation of GFP-DCX immediately before and after the time-lapse sequence, whereas the time-lapse sequence would reveal whether the focal accumulations of GFP-DCX corresponded to axonal domains with abundant protrusive activity and whether they underwent a proximal-to-distal redistribution. We examined 37 axons in this manner. All focal accumulations of GFP-DCX were associated with lamellipodia. In 15 cases, the focal accumulation of DCX underwent little or no change in position during the $10 \mathrm{~min}$ of imaging, and these also exhibited relatively little lamellar activity. However, the remaining 22 exhibited a clear proximal-to-distal redistribution, with 21 advanc- been shown to enhance, retard, or have no effect on axonal growth (He et al., 2005; Qiang et al., 2006; Yu et al., 2008). There is thus ample precedent that the neurons tolerate this manipulation well and can grow vigorously and that this culture regimen is not in any way cytotoxic. Thus, when used in combination with siRNA approaches to manipulate protein levels in the neurons, it provides an effective experimental strategy to explore the functions of these proteins.

Immunostaining and immunoblotting revealed that the DCX siRNA treatment resulted in significant decreases in DCX in both sympathetic and hippocampal neurons after $3 \mathrm{~d}$. The effect was relatively uniform in cultures of sympathetic neurons, with only trace amounts of DCX detected by immunoblotting, and most of the DCX siRNA-treated cells exhibited no detectable DCX stain- 

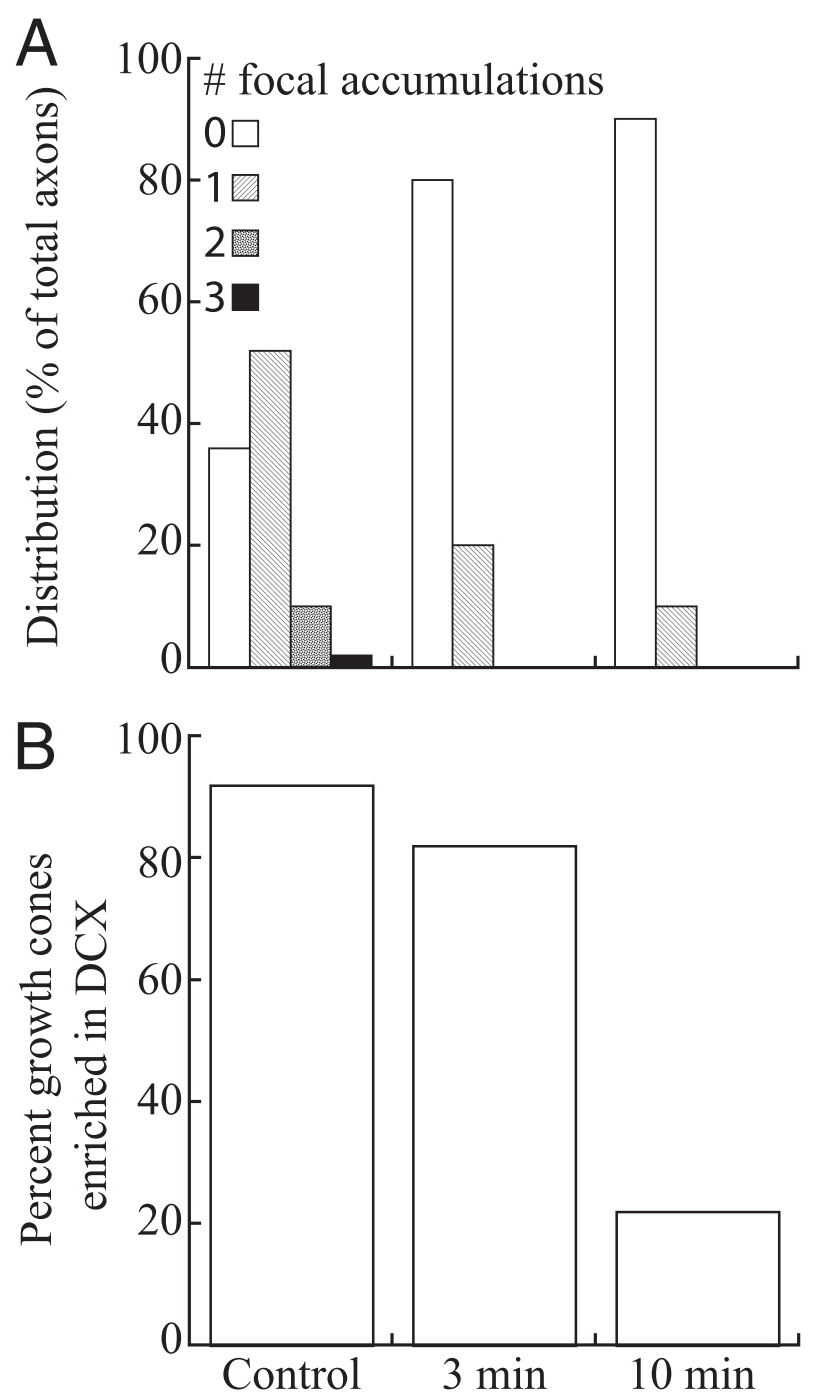

Figure 9. Quantitative analyses of the effects of latrunculin on focal accumulations of DCX along the axon and in the growth cone. $A$ shows frequency distributions of the number of focal accumulations of $D C X$ per axon in control axons and axons treated with latrunculin for 3 or 10 $\min (n=50$ axons per condition). $\boldsymbol{B}$ shows the percentage of growth cones of control and latrunculin-treated neurons that exhibited an enrichment of DCX. This was determined by visual inspection, comparing the staining intensity of the distal region of the axon with the region immediately proximal to it. Growth cones were scored as having an enrichment of DCX if their staining intensity increased abruptly from that of the axonal shaft.

ing above background; background was determined by staining control neurons with a non-immune antibody in place of the DCX antibody. In cultures of hippocampal neurons, immunoblotting also indicated a significant decrease in DCX, but immunostaining revealed that depletion was variable among the neurons. Specifically, $16-31 \%$ of the neurons were very well depleted, having no detectable staining for DCX, 41-47\% were well depleted, but exhibited dim staining that was just above background, and the remaining $28-37 \%$ were poorly depleted in that they clearly stained for DCX, although generally the staining was notably less than that seen in controls (Table 1).

\section{Axonal growth and branching by cultured sympathetic neurons are unaffected by DCX depletion}

Cultured sympathetic neurons are notable for extending axons vigorously in culture and branching extensively by the processes of growth cone bifurcation. DCX depletion had little discernable
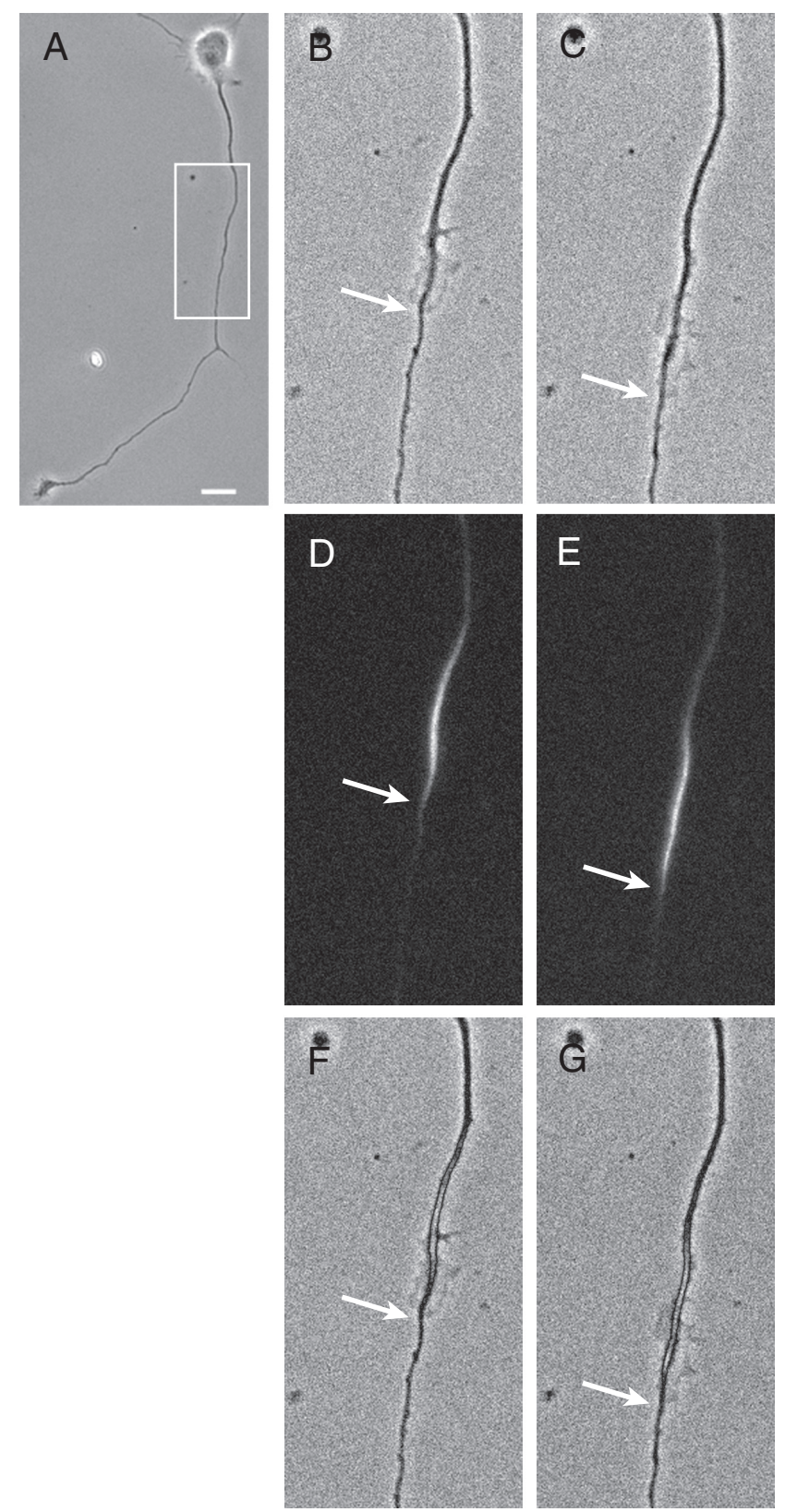

Figure 10. Focal accumulations of $D C X$ undergo a proximal-to-distal redistribution within the axon. $\boldsymbol{A}$ is a low-magnification view of the neuron. The region outlined by the box was evaluated by time-lapse imaging (see supplemental Movie 1, available at www.jneurosci.org as supplemental material). The movie was $10 \mathrm{~min}$ long, with frames taken every $2 \mathrm{~s}$. $B$ and $C$ show the first and last frames of the time-lapse sequence, corresponding to $t=0 \mathrm{~min}$ and $t=10 \mathrm{~min}$, respectively. $\boldsymbol{D}$ shows a fluorescent image of GFP-DCX captured immediately before starting the time-lapse sequence, whereas $\boldsymbol{E}$ shows an image of GFP-DCX taken immediately after the end of the movie. $\boldsymbol{F}$ and $\boldsymbol{G}$ show the fluorescence images overlaid on the phase images. The arrows show the distal extent of the lamellipodia and the GFP-DCX. For additional details, see Results. Scale bar: $\boldsymbol{A}, 14 \mu \mathrm{m} ; \boldsymbol{B}-\mathbf{G}, 9 \mu \mathrm{m}$.

effect on the morphology of cultured sympathetic neurons. By visual inspection, the DCX-depleted neurons robustly extended axons that branched extensively, and in these regards they were not obviously different from control neurons. Quantitative analyses confirmed these impressions. For example, at $\sim 24 \mathrm{~h}$ after replating, the length of the longest axon was $456 \pm 110 \mu \mathrm{m}$ (mean $\pm \mathrm{SD} ; n=23$ axons) and $425 \pm 103 \mu \mathrm{m}(n=25$ axons) in control and DCX-depleted neurons, respectively, values that are statistically indistinguishable $(p>0.1, t$ test). Similarly, total 
Table 1. Effects of DCX depletion on development of hippocampal neurons in culture

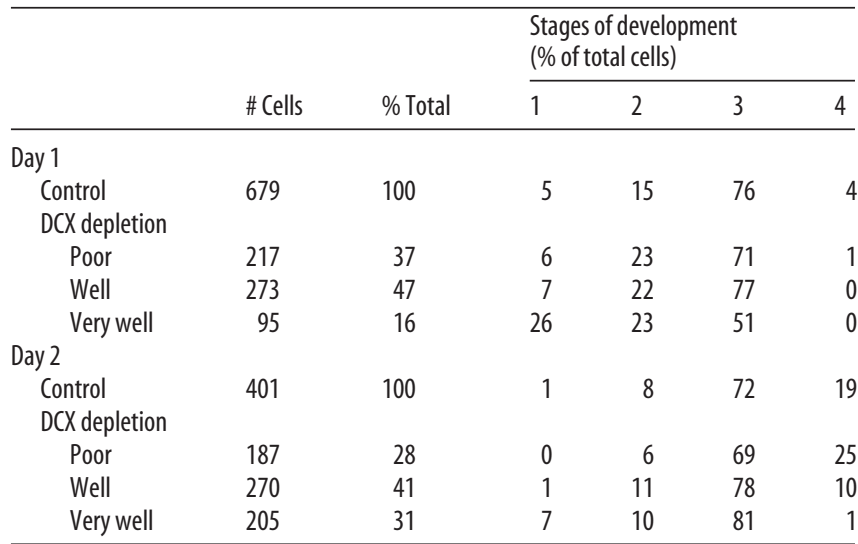

Neurons treated with control siRNA or DCX siRNA were cultured for $3 \mathrm{~d}$, replated as described in Materials and Methods, fixed 1 or $2 \mathrm{~d}$ later, and stained to reveal microtubules and DCX. All cells in representative cultures treated with control siRNA or DCX siRNA were assigned to developmental states 1- 4 according to Dotti et al. (1988). The three categories for the extent of DCX suppression are described in the Results.

branch points per axon, $4.7 \pm 3.8(n=113$ axons $)$ and $4.9 \pm 4.3$ ( $n=102$ axons) for control and DCX-depleted neurons, respectively, were also statistically indistinguishable $(p>0.1, t$ test). Also, the general patterns of staining for actin and microtubules in DCX-depleted cells were not notably distinguishable from that seen in control neurons. The only effect of the DCX siRNA treatment that we detected concerned tau. Whereas the very distal ends of microtubules in growth cones normally stain strongly for DCX but very weakly tau (see above), after DCX depletion, tau staining of the distal portion of microtubules in the growth cone increases dramatically (data not shown) (see also Fig. 12). These latter findings suggest that tau is capable of binding to the microtubule polymer in the peripheral domain of the growth cone, but that the presence of DCX on this polymer normally prevents this from occurring.

\section{DCX depletion from cultured hippocampal neurons retards development, delays axonal branching, and decreases the number of focal actin accumulations along the axonal shaft} Cultured hippocampal neurons undergo a relatively stereotyped sequence of morphological changes during development in culture (Dotti et al., 1988). This progression is slightly accelerated after replating (Table 1, Control) compared with that described for freshly plated cells. Table 1 also shows the development of DCX siRNA-treated neurons, separating the results for cells that were very well depleted, well depleted, and poorly depleted. Cells that had detectable DCX remaining by immunostaining were indistinguishable from controls in terms of the progression through the stages of development. However, the very well depleted cells, which had no detectable DCX staining above background, were slightly retarded in their development in culture. Although many cells were in stage 3 at $1 \mathrm{~d}$ after replating, many more remained in stages 1 and 2 compared with controls. By $2 \mathrm{~d}$ after replating, most of the cells progressed to stage 3 and had relatively long well developed axons. However, compared with controls, many fewer of the very well depleted cells had obvious dendritic processes (Table 1), consistent with recent reports that depletion of DCX delays dendritic development by cultured hippocampal neurons (Deuel et al., 2006; Cohen et al., 2008).

Because of the heterogeneity in the response of hippocampal neurons to treatment with DCX siRNA, we selected for more detailed analyses only those cells that exhibited no detectable staining for DCX. The only other selection criteria used in these analyses was that the cells must be isolated from their neighbors so that there was no possibility of fasciculation of axons from neighboring cells. Although depletion of DCX modestly slows development, those neurons in stage 3 after $1 \mathrm{~d}$ in culture have typical morphology characterized by a single axon and multiple minor processes. By visual inspection, the axons of DCX siRNAtreated cells closely resemble those of controls, and measures of axonal length revealed comparable lengths for control and DCXdepleted cells (Table 2). However, the degree of branching was reduced by DCX depletion (Table 2). The magnitude of this effect was greater at $1 \mathrm{~d}$ after replating compared with $2 \mathrm{~d}(\sim 43$ vs $\sim 32 \%$ ) (Table 2 ). This together with the fact that considerable branching still occurs after DCX depletion indicates that the impact of DCX depletion is to delay branch formation. The results on axonal length and branching indicate that the length of each axon plus all of its branches will on average be somewhat greater for control neurons compared with DCX-depleted neurons. This is consistent with previous reports indicating that DCX depletion modestly reduces the length of axons produced by CNS neurons in culture (Deuel et al., 2006).

The only other notable effect of DCX depletion on the morphology of hippocampal neurons concerned dendrites, which were much less apparent in DCX-depleted cultures compared with controls, especially at $2 \mathrm{~d}$ after plating (Table 1 ). We have not pursued effects of DCX on dendritic development for the present studies, although as indicated above, this result is expected based on recent reports examining the contribution of DCX to dendritic development (Deuel et al., 2006; Cohen et al., 2008).

The formation of axonal branches by hippocampal neurons occurs via the process of collateral branching. Early steps in this process involve a local reorganization of actin filaments and microtubules, leading to the generation of an actin-based protrusive structure that can become transformed into an axonal branch if it is invaded by microtubules (Yu et al., 1994, 2008; Dent et al., 1999). DCX is a bona fide microtubule-associated protein, and it can also interact directly and indirectly with actin filaments. Thus, knockdown of DCX may delay collateral axonal branching by impacting the cytoskeletal reorganizations required to initiate and/or sustain branching. In support of this possibility, we found that DCX depletion also reduced the abundance and appearance of the focal accumulations of actin along the axon. At $1 \mathrm{~d}$ in culture, $\sim 56 \%$ of axons have one or more focal accumulations of actin along the axon, and this increases to $\sim 80 \%$ by $2 \mathrm{~d}$ in culture. DCX reduces this value to $\sim 33 \%$ at both time points (Table 3 ). We also evaluated the average number of focal accumulations of actin in control and DCX-depleted neurons. Comparing control and DCX-depleted axons that contain focal accumulations of actin, the average number per axon is similar in both groups (Table 3). However, the average value is significantly reduced by DCX depletion when computed for the entire population of axons [i.e., those with and without focal accumulations (Table 3)], and this is attributable to the fact that many more DCX-depleted cells lack focal accumulations of actin entirely. In addition, the character of the focal accumulations is altered by DCX depletion. As indicated above, two general categories of focal accumulations can be distinguished, those with or without obvious lamellipodia, with $\sim 40 \%$ having obvious lamellipodia in control neurons. In DCX-depleted neurons, only $\sim 10 \%$ of the focal accumulations have obvious lamellipodia. This difference is statistically highly significant (Table 3). Figure 11 shows a graphical representation of this effect derived from pooling the data from all of the individual experiments included in Table 3; focal actin accumula- 
Table 2. Effects of $D C X$ depletion on axonal length and branching of hippocampal neurons in culture

\begin{tabular}{|c|c|c|c|c|c|c|}
\hline & \multirow{2}{*}{\multicolumn{2}{|c|}{$\begin{array}{l}\text { Experiment } 1 \\
\text { Day } 1 \\
\end{array}$}} & \multicolumn{4}{|l|}{ Experiment 2} \\
\hline & & & \multicolumn{2}{|l|}{ Day 1} & \multicolumn{2}{|l|}{ Day 2} \\
\hline & Control & Depleted & Control & Depleted & Control & Depleted \\
\hline Axonal length $(\mu \mathrm{m})$ & $199 \pm 98(23)^{*}$ & $212 \pm 105(31)^{*}$ & $191 \pm 70(33)^{*}$ & $169 \pm 71(38)^{*}$ & & \\
\hline Branch points/axon & $1.14 \pm 1.5(140)^{\dagger}$ & $0.65 \pm 1.1(134)^{\dagger}$ & $0.62 \pm 1.0(141)^{\ddagger}$ & $0.30 \pm 0.76(127)^{\ddagger}$ & $1.02 \pm 1.5(111)^{\S}$ & $0.69 \pm 1.2(116)^{\S}$ \\
\hline
\end{tabular}

Table 3. Effects of DCX depletion on focal accumulations of actin in axons of hippocampal neurons

\begin{tabular}{|c|c|c|c|c|c|c|c|c|c|}
\hline & \multirow{2}{*}{\multicolumn{3}{|c|}{ Experiment 1}} & \multicolumn{6}{|l|}{ Experiment 2} \\
\hline & & & & \multicolumn{3}{|l|}{ Day 1} & \multicolumn{3}{|l|}{ Day 2} \\
\hline & Control & Depleted & $p$ & Control & Depleted & $p$ & Control & Depleted & $p$ \\
\hline Axons with $\geq 1$ focal accumulation ${ }^{a}$ & $79(56 \%)$ & $43(32 \%)$ & * & $80(56 \%)$ & $39(31 \%)$ & * & $93(80 \%)$ & $38(33 \%)$ & * \\
\hline $\begin{array}{l}\text { Focal accumulations of actin per axon for axons } \\
\text { with focal accumulations }{ }^{b}\end{array}$ & $1.4 \pm 0.7(79)$ & $1.3 \pm 0.6(43)$ & $\dagger$ & $1.5 \pm 0.7(80)$ & $1.5 \pm 0.7(39)$ & $\dagger$ & $1.8 \pm 1(93)$ & $1.9 \pm 1(38)$ & $\dagger$ \\
\hline Focal accumulations of actin per axon for all axons $s^{b}$ & $1.2 \pm 0.8(140)$ & $0.5 \pm 0.7(134)$ & $\ddagger$ & $0.8 \pm 0.9(141)$ & $0.5 \pm 0.8(127)$ & $\ddagger$ & $1.4 \pm 1.2(111)$ & $0.6 \pm 1(116)$ & $\ddagger$ \\
\hline Focal accumulations with lamellipodiac & $49(43 \%)$ & $9(16 \%)$ & $\S$ & $44(37 \%)$ & $4(7 \%)$ & $\S$ & $62(37 \%)$ & $4(6 \%)$ & $\S$ \\
\hline Focal accumulations without lamellipodiac & $64(57 \%)$ & $47(84 \%)$ & $\S$ & $74(63 \%)$ & $53(93 \%)$ & $\S$ & $105(63 \%)$ & 67 (94\%) & $\S$ \\
\hline
\end{tabular}

${ }^{a}$ Data are shown as the total, with the percentage of total in parentheses.

${ }^{b}$ Data are shown as mean $\pm S D$, with $n$ values in parentheses.

'Data are shown as the total, with the percentage of total in parentheses.

${ }^{*} p<0.0001$ ( $\chi^{2}$ test) ${ }^{\dagger} p>0.1$ (Student's $t$ test) $;^{\ddagger} p<0.0001$ (Student's $t$ test) ${ }^{\S} p \leq 0.0005$ ( $\chi^{2}$ test).

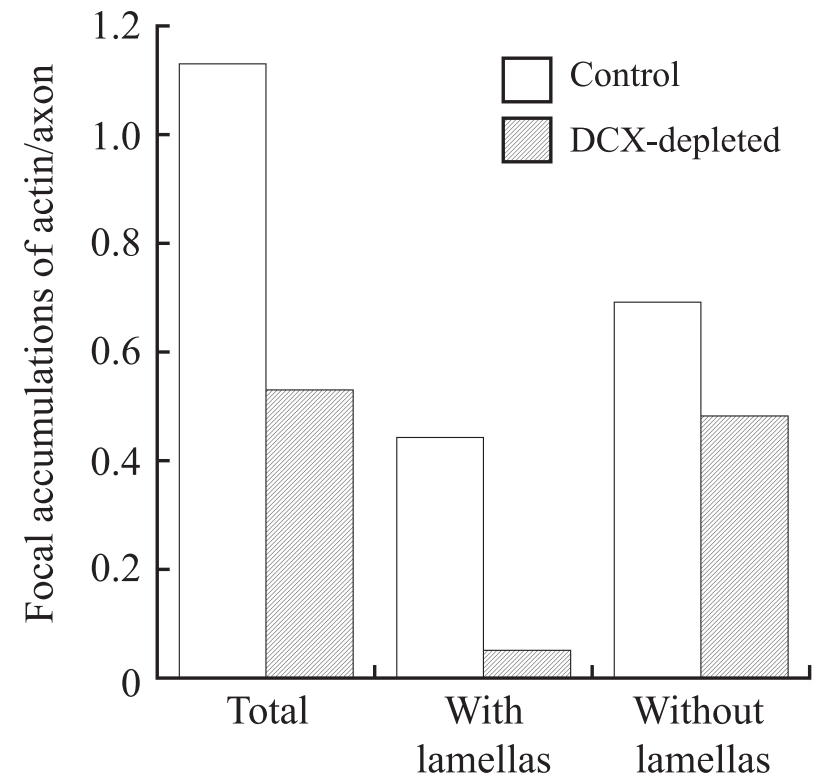

Figure 11. Quantitative analyses of the effects of $D C X$ depletion on the abundance of focal actin accumulations in axons of hippocampal neurons. The data from the individual experiments described in Table 3 were pooled together to generate this graph, which shows the total number of focal accumulations of actin per axon of control or DCX-depleted cells, and the number of focal actin accumulations with or without lamellipodia per axon of control or DCXdepleted cells. DCX depletion reduces the frequency of focal actin accumulations with lamellapodia by $\sim 90 \%$, whereas focal actin accumulations without obvious lamellipodia are reduced by $\sim 30 \%$.

tions with lamellipodia are reduced by $90 \%$, whereas those without obvious lamellipodia are reduced by $\sim 30 \%$.

Actin filament containing structures are also very prominent in growth cones, and this is also true in DCX-depleted cells. We detected no difference in the relative abundance of growth cones with prominent actin filament staining in control and DCXdepleted cells; in both groups, $73 \%$ of the growth cones stained strongly for actin filaments. The many images we captured of growth cone staining for actin filaments and microtubules do not reveal any consistent differences in overall morphology nor in actin filament and microtubule organization that correlate with DCX depletion. However given the natural variation in growth cone morphologies combined with their three-dimensional structure, conventional immunofluorescence imaging could easily miss differences attributable to DCX depletion. The one effect we did observe concerned tau, which, as described above for cultured sympathetic neurons, stained the distal portions of growth cone microtubules much more prominently in DCXdepleted cells compared with controls (Fig. 12).

\section{Overexpression of DCX does not detectably alter the} frequency of focal actin accumulations or of branching As discussed above, depleting DCX reduces the abundance of focal actin accumulations and also delays the formation of collateral axonal branches. To further explore these relationships, we examined the effects of DCX overexpression on hippocampal neurons, specifically testing whether overexpressing DCX will lead to a gain of function manifested as an increase in focal actin accumulations and/or collateral branching. Neurons transfected with constructs encoding GFP only or GFP-DCX were cultured for $3 \mathrm{~d}$ and then fixed and stained for GFP, tubulin, and actin filaments. Images of the cells were then analyzed for total axonal length, the degree of axonal branching, and the abundance of focal actin accumulations. DCX-expressing cells were divided into two groups, low and high expressors. In low expressing cells, GFP-DCX was concentrated in discrete foci along the axon that corresponded with prominent actin containing lamellas (Fig. 10) (supplemental Fig. 4, available at www.jneurosci.org as supplemental material). In high expressing cells, GFP-DCX was present at relatively high levels along the entire length of the axon. Focal accumulations of actin were present at discrete sites in the axons of many of these cells, and all of these were associated with relatively strong staining for GFP-DCX. In some axons, the GFPDCX was more concentrated at sites of focal actin accumulations 

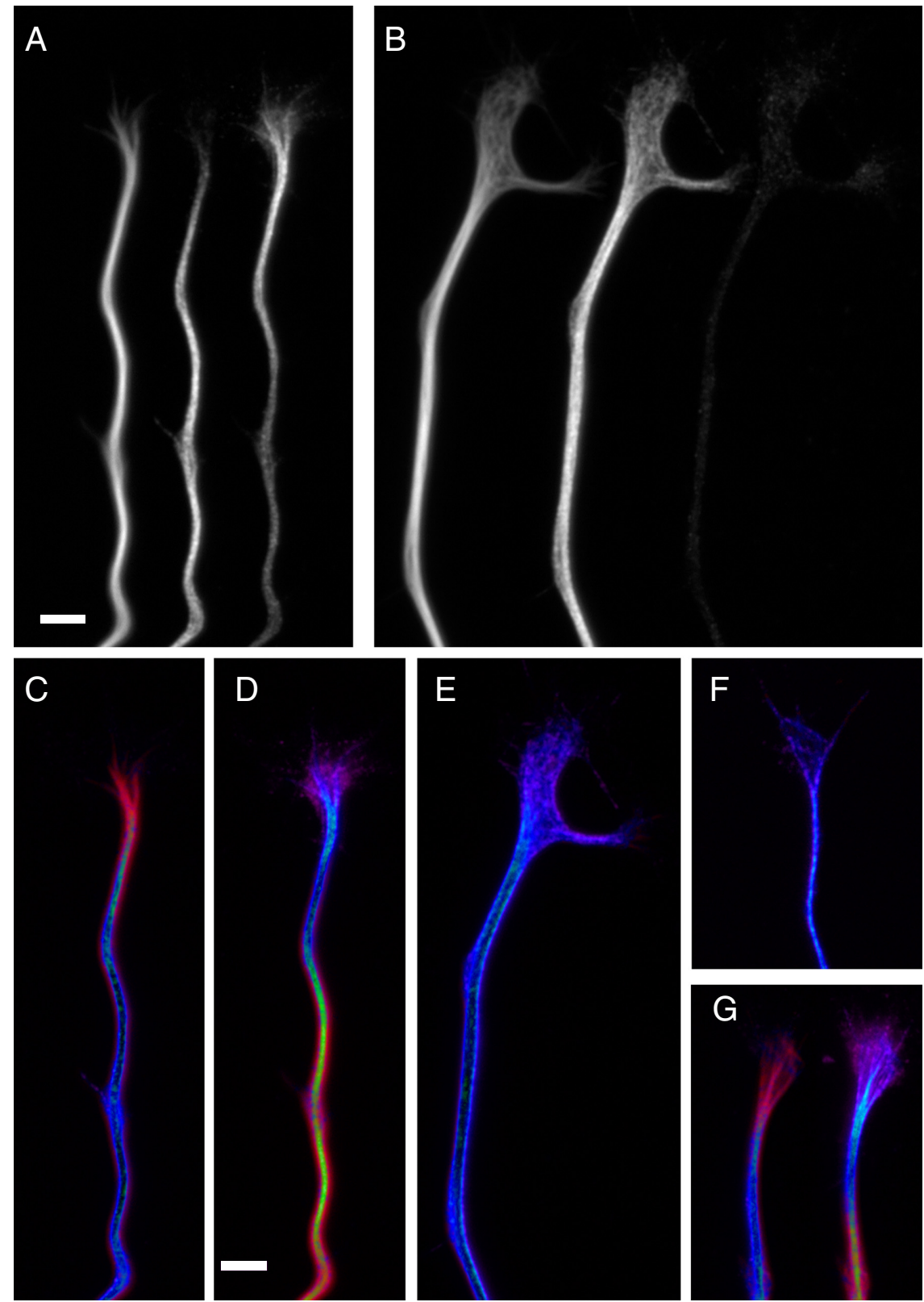

Figure 12. DCX normally displaces tau from microtubules in the distal parts of the growth cone. Hippocampal neurons treated with control or DCX siRNA for $3 \mathrm{~d}$ were replated and allowed to extend axons for $\sim 1 \mathrm{~d}$, at which time they were fixed and then triple stained to reveal microtubules, DCX, and tau. $\boldsymbol{A}$ and $\boldsymbol{B}$ show, from left to right, grayscale images of microtubules, tau, and $D C X$ staining in the distal axon and growth cone from cells treated with control siRNA $(\boldsymbol{A})$ or DCX siRNA $(\boldsymbol{B})$. $\boldsymbol{C}-\boldsymbol{G}$ show overlay images in which microtubule staining is depicted in red, whereas tau or DCX staining is depicted in violet; areas of overlap appear as blue in such overlays. Cand $\boldsymbol{D}$ depict tau/microtubule and DCX/microtubule overlays, respectively, from the control neuron shown in $\boldsymbol{A}$, whereas $\boldsymbol{E}$ shows the tau/microtubule overlay from the $D C X$-depleted cell depicted in $\boldsymbol{B}$. $\boldsymbol{F}$ shows a tau/microtubule overlay from a different $D C X$-depleted cell, whereas $\mathbf{G}$ shows tau/microtubule and DCX/microtubule overlays (left and right, respectively) from a different control siRNA-treated neuron. In all control neurons, DCX staining is prominent in the growth cone, decorating the microtubules therein to their distal extents, whereas tau staining is strongest in the distal axon and is relatively weak on growth cone microtubules. In DCX-depleted cells, tau staining is strong on microtubules throughout the growth cone. Scale bars: $A($ for $A, B), D($ for $(-G)$, $3.5 \mu \mathrm{m}$.

relative to adjacent axonal regions, whereas in other axons, GFPDCX did not appear preferentially concentrated at the focal actin accumulations compared with adjacent axonal regions (supplemental Fig. 4, available at www.jneurosci.org as supplemental material). As shown in Table 4, cells strongly expressing GFPDCX were statistically indistinguishable from low expressing cells and from cells overexpressing GFP in axonal length, axonal
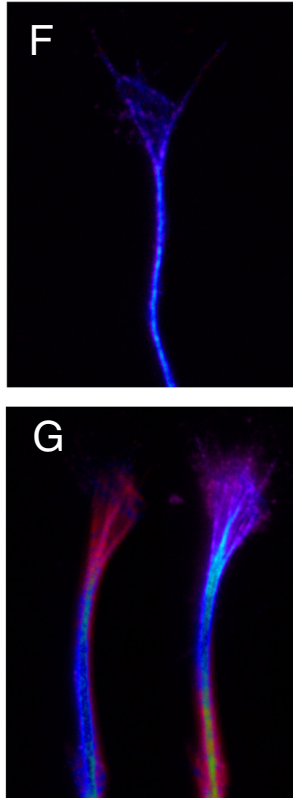

branching, and the abundance of focal actin accumulations. Thus, augmenting DCX alone is not sufficient to affect the abundance of focal actin accumulations or collateral branches.

\section{Discussion}

We have identified the transition zone and peripheral domain of the growth cone as key sites of DCX enrichment in growing axons. This distribution is not consistent with the recent proposal that DCX functions chiefly in the neck (or "wrist") of the growth cone, in which it has been speculated to promote microtubule bundling and thereby contribute to axonal consolidation (Bielas et al., 2007). Given that DCX depletion retards the timetable of development of cultured hippocampal neurons, it may be that the observations made by the earlier authors on microtubule spacing may have been made on immature processes rather than bona fide axons. Their own images actually support this view, with the processes from DCX-deficient neurons displaying elongated lamellae characteristic of earlier developmental stages. They also examined older DCX-depleted neurons, and these had definitive axons with no indication of abnormal splaying indicative of impaired microtubule bundling. We also did not detect enhanced splaying of microtubules in axons of DCX-deficient neurons. Finally, the promotion of microtubule bundling by DCX has only been observed at very high stoichiometries of DCX relative to tubulin (Horesh et al., 1999; Moores et al., 2006). Thus, if DCX functions to bundle microtubules in the growth cone neck, we would surmise that it should be present in this region at high stoichiometries relative to tubulin. Our ratio imaging shows that the stoichiometry of DCX on microtubules is actually much higher in the distal growth cone compared with the neck region. If the stoichiometry in the neck is sufficient to induce significant microtubule bundling, then even more bundling should occur more distally in the growth cone. Contrary to this, the microtubule polymer in the growth cone that is richest in DCX and thus has the highest stoichiometry is not bundled at all. Instead, the microtubules are splayed and often widely separated from each other. Thus, rather than functioning at the neck of the growth cone, it would appear that DCX functions mainly at the opposite end of the growth cone, in which microtubules splay and engage the actin cytoskeleton. This view is consistent with observations that DCX can interact with both microtubules and actin filaments (Tsukada et al., 2003, 2005, 2006; Shmueli et al., 2006). 
Table 4. Overexpression of $D C X$ does not detectably affect axonal branching or the abundance of focal accumulations

\begin{tabular}{llll}
\hline & $\begin{array}{l}\text { Branches } \\
\text { per } 100 \mu \mathrm{m}\end{array}$ & $\begin{array}{l}\text { Focal accumulations } \\
\text { per } 100 \mu \mathrm{m}\end{array}$ & $\begin{array}{l}\text { Total axon } \\
\text { length }(\mu \mathrm{m})\end{array}$ \\
\hline $\begin{array}{l}\text { GFP expressers }(n=92) \\
\text { DCX expressers }\end{array}$ & $0.42 \pm 0.05$ & $0.57 \pm 0.06$ & $322 \pm 15$ \\
Low $(n=61)$ & $0.32 \pm 0.05$ & $0.66 \pm 0.07$ & $280 \pm 16$ \\
High $(n=90)$ & $0.40 \pm 0.05$ & $0.70 \pm 0.05$ & $290 \pm 12$ \\
\hline
\end{tabular}

Dissociated neurons were transfected with plasmids for GFP or GFP-DCX, cultured for $3 \mathrm{~d}$, and then fixed and stained for GFP, actin, and tubulin. Images were obtained from each cell that was well isolated from its neighbors. The tubulin images were used to quantify the length of the axon, including all of its branches, and the actin images were used to quantify focal accumulations. For the latter, we only counted those with obvious lamellipodia. Data shown are means \pm SEs. DCX expression was evaluated by visual inspection; low expressers exhibited little to no GFP staining, whereas high expressers exhibited obvious staining for GFP that filled the cell body and the entire axon. Data were compared statistically using Student's $t$ test, and in all cases $p>0.06$

Whatever the specific functions of DCX in the growth cone, our data suggest that it is not essential for axonal growth per se because its depletion has only modest affects on axonal growth (Deuel et al., 2006). This may reflect the activities of other proteins in the growth cone that can compensate for the loss of DCX, such as DCX-like kinase (Burgess and Reiner, 2000; Lin et al., 2000; Kim et al., 2003; Edelman et al., 2005; Deuel et al., 2006; Shu et al., 2006). It also seems reasonable that DCX may have unique functions in axonal morphogenesis, but that revealing these functions will require assays that examine more subtle features than simply the extent of axonal growth. For example, DCX seems well suited to participate in the steering of the growth cone, by virtue of its location and ability to interact with both microtubules and actin filaments, as well as its ability to interact with scaffolding proteins for signal transduction pathways (Tsukada et al., 2003, 2005, 2006; Gdalyahu et al., 2004; Shmueli et al., 2006). The fact that the microtubule polymer richest in DCX is also relatively deficient in tau may be important for regulating events such as the severing of microtubules, given that microtubules deficient in tau are more sensitive to severing by katanin (Qiang et al., 2006; Yu et al., 2008).

In sympathetic neurons, little DCX is present along the axonal shaft, whereas in hippocampal neurons, enrichments of DCX occurred at discrete sites along the axon. The regions rich in DCX are rich in actin filaments, exhibit vigorous filopodial and lamellipodial protrusive activity, and undergo a proximal-to-distal redistribution over time. In all of these respects, they are identical to the "growth cone like waves" originally described by Ruthel and Banker (1998, 1999). These authors also showed that the waves are rich in GAP43 and ERM proteins, proteins important in growth cone function. In a more recent study published on these waves, Flynn et al. (2009) confirmed their observations and identified several additional proteins in waves that are also enriched in growth cones.

Given their striking resemblance to growth cones in so many respects, it seems reasonable to suggest that, like growth cones, these waves are specialized to generate motile behaviors that help sculpt the shape of the axon. Flynn et al. (2009) showed that the minor process of hippocampal neurons that will become the axon begins to exhibit a higher frequency of waves shortly before undergoing the burst of growth that identifies it as the axon, and the increased frequency of waves apparently contributes to this burst of growth. They also showed that waves can initiate collateral branch formation by entering filopodia along the axonal shaft and promoting their elongation and stabilization. If waves normally facilitate these processes, then manipulations that reduce the frequency of waves should delay both of these processes. In support of this, we showed that depleting DCX reduces the occurrence of focal actin accumulations, and it is the focal actin accumulations that have prominent lamellipodia that are most significantly affected (Fig. 11). Because these correspond to the waves, we conclude that depleting DCX reduces the frequency of waves, and concomitantly delays the differentiation of the axon (Table 1), and also delays the formation of collateral branches (Table 2).

Additional correlative evidence linking the waves to collateral branching is the fact that axons of sympathetic neurons lack focal accumulations of DCX and actin filaments, and we assume also lack waves, and similarly undergo little or no collateral branching. We note that depleting DCX in hippocampal neurons reduces the occurrence of waves but does not eliminate them entirely and, similarly, delays collateral branching but does not suppress it completely. The waves remaining after DCX depletion may be sufficient to sustain the branching that occurs under these conditions, or, as discussed by Flynn et al. (2009), sometimes axonal differentiation and collateral branching occur without detecting any waves at all, indicating that, although waves can promote these processes, they are not essential for their occurrence. Finally, it is curious that the leading process of migratory neurons depleted of DCX actually undergo increased branching (Kappeler et al., 2006; Friocourt et al., 2007), indicating that DCX can function rather differently in the process of branching in different contexts (Bielas et al., 2007).

One of the novel findings reported here is the striking association of DCX with microtubules preferentially in regions rich in actin filaments. The focal concentrations of DCX in the waves and growth cone are lost after acute depolymerization of actin filaments. This result was surprising given that the majority of the DCX localizes with microtubules and not actin filaments. In this regard, components of various signaling pathways can interact with actin filaments in a manner that influences their activity. It may be that acute actin depolymerization alters the activity of these pathways with a downstream affect on DCX that affects its association with microtubules (Fukata et al., 2003; Schaar et al., 2004; Pullikuth and Catling 2007). It is also noteworthy that depleting DCX reduces the frequency of occurrence of the growthcone-like waves. This indicates a fundamental role for DCX in the generation and/or maintenance of waves. In this regard, DCX has binding activities for microtubules, actin filaments, and a variety of other proteins (Friocourt et al., 2001, 2005; Kizhatil et al., 2002; Tsukada et al., 2003, 2005; Gdalyahu et al., 2004; Reiner et al., 2006), raising the possibility that it may function as a component of a scaffold to bring together components necessary to form the waves. Neurabin II, an actin-binding protein that also can bind DCX (Tsukada et al., 2003, 2005, 2006; Shmueli et al., 2006), may also be a component of this scaffold because it is also present in the waves (D. Jean and M. M. Black, unpublished data).

At the onset of these studies on doublecortin, we had not expected to rediscover the growth-associated waves originally observed over 10 years ago but rarely discussed in the literature since then. The recent work by Flynn et al. (2009) together with the data reported here reveal the waves as highly sophisticated structures capable of boosting the growth of the axon as well as newly forming collateral branches. The entry of a wave into a growth cone or a filopodia may enhance the sensitivity to local morphogens and also provide cytoskeletal components that fuel a burst of elongation. In this view, the waves function as mobile growth boosters that provide growth-promoting materials to sites of impending morphological change. The fact that certain kinds of neurons display these waves whereas others do not may 
be a key determinant of the distinct growth behaviors and morphologies exhibited by these different types of neurons.

\section{References}

Bielas SL, Serneo FF, Chechlacz M, Deerinck TJ, Perkins GA, Allen PB, Ellisman MH, Gleeson JG (2007) Spinophilin facilitates dephosphorylation of doublecortin by PP1 to mediate microtubule bundling at the axonal wrist. Cell 129:579-591.

Black MM, Slaughter T, Moshiach S, Obrocka M, Fischer I (1996) Tau is enriched on dynamic microtubules in the distal region of growing axons. J Neurosci 16:3601-3619.

Blose SH, Meltzer DI, Feramisco JR (1984) 10-nm filaments are induced to collapse in living cells microinjected with monoclonal and polyclonal antibodies against tubulin. J Cell Biol 98:847-858.

Bouquet C, Ravaille-Veron M, Propst F, Nothias F (2007) MAP1B coordinates microtubule and actin filament remodeling in adult mouse Schwann cell tips and DRG neuron growth cones. Mol Cell Neurosci 36:235-247.

Brown A, Slaughter T, Black MM (1992) Newly assembled microtubules are concentrated in the proximal and distal regions of growing axons. J Cell Biol 119:867-882.

Burgess HA, Reiner O (2000) Doublecortin-like kinase is associated with microtubules in neuronal growth cones. Mol Cell Neurosci 16:529-541.

Cohen D, Segal M, Reiner O (2008) Doublecortin supports the development of dendritic arbors in primary hippocampal neurons. Dev Neurosci 30:187-199.

Dent EW, Gertler FB (2003) Cytoskeletal dynamics and transport in growth cone motility and axon guidance. Neuron 40:209-227.

Dent EW, Kalil K (2001) Axon branching requires interactions between dynamic microtubules and actin filaments. J Neurosci 21:9757-9769.

Dent EW, Callaway JL, Szebenyi G, Baas PW, Kalil K (1999) Reorganization and movement of microtubules in axonal growth cones and developing interstitial branches. J Neurosci 19:8894-8908.

Deuel TA, Liu JS, Corbo JC, Yoo SY, Rorke-Adams LB, Walsh CA (2006) Genetic interactions between doublecortin and doublecortin-like kinase in neuronal migration and axon outgrowth. Neuron 49:41-53.

Dotti CG, Sullivan CA, Banker GA (1988) The establishment of polarity by hippocampal neurons in culture. J Neurosci 8:1454-1468.

Edelman AM, Kim WY, Higgins D, Goldstein EG, Oberdoerster M, Sigurdson W (2005) Doublecortin kinase-2, a novel doublecortin-related protein kinase associated with terminal segments of axons and dendrites. J Biol Chem 280:8531-8543.

Flynn KC, Pak CW, Shaw AE, Bradke F, Bamburg JR (2009) Growth conelike waves transport actin and promote axonogenesis and neurite branching. Dev Neurobiol. Retrieved June 16, 2009. doi:10.1002/dneu.20734.

Francis F, Koulakoff A, Boucher D, Chafey P, Schaar B, Vinet MC, Friocourt G, McDonnell N, Reiner O, Kahn A, McConnell SK, Berwald-Netter Y, Denoulet P, Chelly J (1999) Doublecortin is a developmentally regulated, microtubule-associated protein expressed in migrating and differentiating neurons. Neuron 23:247-256.

Friocourt G, Chafey P, Billuart P, Koulakoff A, Vinet MC, Schaar BT, McConnell SK, Francis F, Chelly J (2001) Doublecortin interacts with mu subunits of clathrin adaptor complexes in the developing nervous system. Mol Cell Neurosci 18:307-319.

Friocourt G, Koulakoff A, Chafey P, Boucher D, Fauchereau F, Chelly J, Francis F (2003) Doublecortin functions at the extremities of growing neuronal processes. Cereb Cortex 13:620-626.

Friocourt G, Kappeler C, Saillour Y, Fauchereau F, Rodriguez MS, Bahi N, Vinet MC, Chafey P, Poirier K, Taya S, Wood SA, Dargemont C, Francis F, Chelly J (2005) Doublecortin interacts with the ubiquitin protease DFFRX, which associates with microtubules in neuronal processes. Mol Cell Neurosci 28:153-164.

Friocourt G, Liu JS, Antypa M, Rakic S, Walsh CA, Parnavelas JG (2007) Both doublecortin and doublecortin-like kinase play a role in cortical interneuron migration. J Neurosci 27:3875-3883.

Fukata M, Nakagawa M, Kaibuchi K (2003) Roles of Rho-family GTPases in cell polarization and directional migration. Curr Opin Cell Biol 15:590-597.

Gdalyahu A, Ghosh I, Levy T, Sapir T, Sapoznik S, Fishler Y, Azoulai D, Reiner O (2004) DCX, a new mediator of the JNK pathway. EMBO J 23:823-832.
Gleeson JG, Lin PT, Flanagan LA, Walsh CA (1999) Doublecortin is a microtubule-associated protein and is expressed widely by migrating neurons. Neuron 23:257-271.

Grabham PW, Reznik B, Goldberg DJ (2003) Microtubule and Rac 1-dependent F-actin in growth cones. J Cell Sci 116:3739-3748.

Graham ME, Ruma-Haynes P, Capes-Davis AG, Dunn JM, Tan TC, Valova VA, Robinson PJ, Jeffrey PL (2004) Multisite phosphorylation of doublecortin by cyclin-dependent kinase 5 . Biochem J 381:471-481.

Hasaka TP, Myers KA, Baas PW (2004) Role of actin filaments in the axonal transport of microtubules. J Neurosci 24:11291-11301.

He Y, Francis F, Myers KA, Yu W, Black MM, Baas PW (2005) Role of cytoplasmic dynein in the axonal transport of microtubules and neurofilaments. J Cell Biol 168:697-703.

Horesh D, Sapir T, Francis F, Wolf SG, Caspi M, Elbaum M, Chelly J, Reiner O (1999) Doublecortin, a stabilizer of microtubules. Hum Mol Genet 8:1599-1610.

Kaech S, Banker G (2006) Culturing hippocampal neurons. Nat Protoc 1:2406-2415.

Kappeler C, Saillour Y, Baudoin JP, Tuy FP, Alvarez C, Houbron C, Gaspar P, Hamard G, Chelly J, Métin C, Francis F (2006) Branching and nucleokinesis defects in migrating interneurons derived from doublecortin knockout mice. Hum Mol Genet 15:1387-1400.

Kempf M, Clement A, Faissner A, Lee G, Brandt R (1996) Tau binds to the distal axon early in development of polarity in a microtubule-and microfilament-dependent manner. J Neurosci 16:5583-5592.

Kim MH, Cierpicki T, Derewenda U, Krowarsch D, Feng Y, Devedjiev Y, Dauter Z, Walsh CA, Otlewski J, Bushweller JH, Derewenda ZS (2003) The DCX-domain tandems of doublecortin and doublecortin-like kinase. Nat Struct Biol 10:324-333.

Kizhatil K, Wu YX, Sen A, Bennett V (2002) A new activity of doublecortin in recognition of the phospho-FIGQY tyrosine in the cytoplasmic domain of neurofascin. J Neurosci 22:7948-7958.

Lin PT, Gleeson JG, Corbo JC, Flanagan L, Walsh CA (2000) DCAMKL1 encodes a protein kinase with homology to doublecortin that regulates microtubule polymerization. J Neurosci 20:9152-9161.

Mandell JW, Banker GA (1996) A spatial gradient of tau protein phosphorylation in nascent axons. J Neurosci 16:5727-5740.

Moores CA, Perderiset M, Francis F, Chelly J, Houdusse A, Milligan RA (2004) Mechanism of microtubule stabilization by doublecortin. Mol Cell 14:833-839.

Moores CA, Perderiset M, Kappeler C, Kain S, Drummond D, Perkins SJ, Chelly J, Cross R, Houdusse A, Francis F (2006) Distinct roles of doublecortin modulating the microtubule cytoskeleton. EMBO J 25:4448-4457.

Myers KA, Tint I, Nadar CV, He Y, Black MM, Baas PW (2006) Antagonistic forces generated by cytoplasmic dynein and myosin-II during growth cone turning and axonal retraction. Traffic 7:1333-1351.

Pullikuth AK, Catling AD (2007) Scaffold mediated regulation of MAPK signaling and cytoskeletal dynamics: a perspective. Cell Signal 19:1621-1632.

Qiang L, Yu W, Andreadis A, Luo M, Baas PW (2006) Tau protects microtubules in the axon from severing by katanin. J Neurosci 26:3120-3129.

Reiner O, Coquelle FM, Peter B, Levy T, Kaplan A, Sapir T, Orr I, Barkai N, Eichele G, Bergmann S (2006) The evolving doublecortin (DCX) superfamily. BMC Genomics 7:188.

Rivas RJ, Burmeister DW, Goldberg DJ (1992) Rapid effects of laminin on the growth cone. Neuron 8:107-115.

Roy S, Coffee P, Smith G, Liem RK, Brady ST, Black MM (2000) Neurofilaments are transported rapidly but intermittently in axons: implications for slow axonal transport. J Neurosci 20:6849-6861.

Roy S, Winton MJ, Black MM, Trojanowski JQ, Lee VM (2008) Cytoskeletal requirements in axonal transport of slow component-b. J Neurosci 28:5248-5256.

Ruthel G, Banker G (1998) Actin-dependent anterograde movement of growth-cone-like structures along growing hippocampal axons: a novel form of axonal transport? Cell Motil Cytoskeleton 40:160-173.

Ruthel G, Banker G (1999) Role of moving growth cone-like "wave" structures in the outgrowth of cultured hippocampal axons and dendrites. J Neurobiol 39:97-106.

Sankaranarayanan S, Atluri PP, Ryan TA (2003) Actin has a molecular scaffolding, not propulsive, role in presynaptic function. Nat Neurosci 6:127-135. 
Schaar BT, Kinoshita K, McConnell SK (2004) Doublecortin microtubule affinity is regulated by a balance of kinase and phosphatase activity at the leading edge of migrating neurons. Neuron 41:203-213.

Shmueli A, Gdalyahu A, Sapoznik S, Sapir T, Tsukada M, Reiner O (2006) Site-specific dephosphorylation of doublecortin (DCX) by protein phosphatase 1 (PP1). Mol Cell Neurosci 32:15-26.

Shu T, Tseng HC, Sapir T, Stern P, Zhou Y, Sanada K, Fischer A, Coquelle FM, Reiner O, Tsai LH (2006) Doublecortin-like kinase controls neurogenesis by regulating mitotic spindles and $\mathrm{M}$ phase progression. Neuron 49:25-39.

Slaughter T, Wang J, Black MM (1997) Microtubule transport from the cell body into the axons of growing neurons. J Neurosci 17:5807-5819.

Tanaka T, Serneo FF, Tseng HC, Kulkarni AB, Tsai LH, Gleeson JG (2004) Cdk5 phosphorylation of doublecortin ser297 regulates its effect on neuronal migration. Neuron 41:215-227.

Tsukada M, Prokscha A, Oldekamp J, Eichele G (2003) Identification of neurabin II as a novel doublecortin interacting protein. Mech Dev 120:1033-1043.

Tsukada M, Prokscha A, Ungewickell E, Eichele G (2005) Doublecortin association with actin filaments is regulated by neurabin II. J Biol Chem 280:11361-11368.

Tsukada M, Prokscha A, Eichele G (2006) Neurabin II mediates doublecortin-dephosphorylation on actin filaments. Biochem Biophys Res Commun 343:839-847.

Yu W, Ahmad FJ, Baas PW (1994) Microtubule fragmentation and partitioning in the axon during collateral branch formation. J Neurosci 14:5872-5884.

Yu W, Ling C, Baas PW (2001) Microtubule reconfiguration during axogenesis. J Neurocytol 30:861-875.

Yu W, Qiang L, Solowska JM, Karabay A, Korulu S, Baas PW (2008) The microtubule-severing proteins spastin and katanin participate differently in the formation of axonal branches. Mol Biol Cell 19:1485-1498. 